\title{
Do Stable Outcomes Survive in Marriage Problems with Myopic and Farsighted Players?
}

Citation for published version (APA):

Herings, P. J-J., Mauleon, A., \& Vannetelbosch, V. (2020). Do Stable Outcomes Survive in Marriage Problems with Myopic and Farsighted Players? Maastricht University, Graduate School of Business and Economics. GSBE Research Memoranda No. 031 https://doi.org/10.26481/umagsb.2020031

Document status and date:

Published: $16 / 11 / 2020$

DOI:

10.26481/umagsb.2020031

Document Version:

Publisher's PDF, also known as Version of record

\section{Please check the document version of this publication:}

- A submitted manuscript is the version of the article upon submission and before peer-review. There can be important differences between the submitted version and the official published version of record.

People interested in the research are advised to contact the author for the final version of the publication, or visit the DOI to the publisher's website.

- The final author version and the galley proof are versions of the publication after peer review.

- The final published version features the final layout of the paper including the volume, issue and page numbers.

Link to publication

\footnotetext{
General rights rights.

- You may freely distribute the URL identifying the publication in the public portal. please follow below link for the End User Agreement:

www.umlib.nl/taverne-license

Take down policy

If you believe that this document breaches copyright please contact us at:

repository@maastrichtuniversity.nl

providing details and we will investigate your claim.
}

Copyright and moral rights for the publications made accessible in the public portal are retained by the authors and/or other copyright owners and it is a condition of accessing publications that users recognise and abide by the legal requirements associated with these

- Users may download and print one copy of any publication from the public portal for the purpose of private study or research.

- You may not further distribute the material or use it for any profit-making activity or commercial gain

If the publication is distributed under the terms of Article $25 \mathrm{fa}$ of the Dutch Copyright Act, indicated by the "Taverne" license above, 
P. Jean-Jacques Herings, Ana Mauleon, Vincent Vannetelbosch

Do Stable Outcomes Survive in Marriage Problems with Myopic and Farsighted Players?

$\mathrm{RM} / 20 / 031$

ISSN : $2666-8807$

\section{GSBE}

Maastricht University School of Business and Economics

Graduate School of Business and Economics

P.O Box 616

NL- 6200 MD Maastricht

The Netherlands 


\title{
Do Stable Outcomes Survive in Marriage Problems with Myopic and Farsighted Players?
}

\author{
P. Jean-Jacques Herings* Ana Mauleon ${ }^{\dagger}$ \\ Vincent Vannetelbosch ${ }^{\ddagger}$
}

November 2, 2020

\begin{abstract}
We consider marriage problems where myopic and farsighted players interact. To study such problems, we use the pairwise myopic-farsighted stable set. Blocking occurs by coalitions of size one or two. We require that all blocking players should strictly improve. We pay particular attention to the question whether core elements survive in this environment. This is the case when all players are myopic as well as when all players are farsighted. It also holds for matching problems satisfying the top-coalition property. For general matching problems where all women are farsighted, there is only one core element that can belong to the pairwise myopic-farsighted stable set, the woman-optimal stable matching, so all other stable outcomes are excluded for sure. If the woman-optimal stable matching is dominated from the woman point of view by an individually rational matching, then the pairwise myopicfarsighted stable set cannot contain a core element. We show that blocking by coalitions of arbitrary size leads to identical results.

Key words: Marriage problems, core, stable sets, myopic and farsighted players.

JEL classification: C70, C78.
\end{abstract}

*Department of Economics, Maastricht University, Maastricht, The Netherlands. E-mail: P.Herings@maastrichtuniversity.nl

${ }^{\dagger}$ CEREC and CORE, UCLouvain, Belgium. E-mail: ana.mauleon@usaintlouis.be

${ }^{\ddagger}$ CORE and CEREC, UCLouvain, Belgium. E-mail: vincent.vannetelbosch@uclouvain.be 


\section{Introduction}

Stability is considered to be a crucial property in marriage problems. A matching is stable if no individual player prefers to leave his/her current partner and no pair of players prefers to form a match between them. For marriage problems, this stability notion is equivalent to core stability. A matching is in the core if there is no subset of players who can all obtain a strictly preferred outcome by forming only partnerships among themselves. Gale and Shapley (1962) have shown that the set of stable matchings, and therefore the core, is non-empty. Both myopic and farsighted solution concepts point towards the core as the set of reasonable outcomes. Moreover, both approaches are not able to discriminate between different core elements.

Several myopic solution concepts coincide with the core. Indeed, although the core might be a proper subset of the von Neumann-Morgenstern (vNM) stable set (Ehlers, 2007), which is unique by the results in Wako (2010), the equivalence between core and vNM stable set is restored by the reformulation of the vNM stable set in Herings, Mauleon and Vannetelbosch (2017), called the CP vNM set. The core also coincides with the myopic stable set of Demuynck, Herings, Saulle and Seel (2019).

Notions like the core, the vNM stable set, the pairwise CP vNM set, and the myopic stable set are myopic notions since the players do not anticipate that individual and coalitional deviations are countered by subsequent deviations. These concepts are based on the direct dominance relation and neglect the destabilizing effect of indirect dominance relations as introduced by Harsanyi (1974) and Chwe (1994). Indirect dominance captures the idea that farsighted players can anticipate the actions of other coalitions and consider the end matching that their deviations may lead to.

When direct dominance is replaced by indirect dominance in the definition of the vNM stable set, we obtain the vNM farsightedly stable set. ${ }^{1}$ Surprisingly, the

\footnotetext{
${ }^{1}$ Other approaches to farsightedness in coalition and network formation are the largest consistent set of Chwe (1994), the optimistic and conservative stable standards of behavior of Xue (1998), the stable set with respect to path dominance in Page and Wooders (2009) and farsightedly stability as defined in Herings, Mauleon and Vannetelbosch (2009). Non-cooperative approaches towards farsightedness have been used as well. Bloch (1996) studies the stationary subgame perfect equilibria of a dynamic game, whereas Herings, Mauleon and Vannetelbosch (2004) use an
} 
core outcomes are still the most relevant ones. Diamantoudi and Xue (2003) have shown that coalition structures in the core are farsighted stable in hedonic games. For marriage problems, Mauleon, Vannetelbosch, and Vergote (2011) characterize the vNM farsightedly stable sets as all singletons that contain a core element. The equivalence between vNM farsightedly stable sets and singleton core elements actually holds quite generally in characteristic function games as shown in Ray and Vohra (2015). Such results are also robust to the incorporation of various forms of maximality in the indirect dominance relation, like the strong rational expectations farsighted stable set in Dutta and Vohra (2017) and absolute maximality as in Ray and Vohra (2019). ${ }^{2}$

However, the concepts studied so far in the literature do not allow for heterogeneity regarding the degree of farsightedness of the players in the market. In this paper, we allow for the interaction between myopic and farsighted players and propose to analyze this interaction by means of the pairwise myopic-farsighted stable set. A pairwise myopic-farsighted stable set is the set of matchings satisfying internal and external stability with respect to the notion of a pairwise myopic-farsighted improving path. That is, there is no pairwise myopic-farsighted improving path from any matching in the set to another matching in the set (internal stability) and there is a pairwise myopic-farsighted improving path from any matching outside the set to some matching in the set (external stability).

We define a pairwise myopic-farsighted improving path as a sequence of matchings that can emerge when farsighted players form or destroy matches based on the improvement the end matching offers relative to the current one while myopic players form or destroy matches based on the improvement the next matching offers relative to the current one. Each matching in the sequence differs from the previous one in that either a new match is formed or an existing one is destroyed. If a match approach based on rationalizability. Anesi (2010) supports the vNM stable set as a stationary subgame perfect equilibrium in legislative bargaining games. The dynamic process of coalition formation by Konishi and Ray (2003) has many features of a non-cooperative equilibrium and can be obtained as a non-cooperative equilibrium if one allows the process to be history-dependent as shown in Vartiainen (2011). Kimya (2020) proposes the equilibrium coalitional behavior and the credible equilibrium coalitional behavior and bridges the non-cooperative and the cooperative approaches to farsightedness.

${ }^{2}$ Recent research along these lines allows for heterogeneous expectations in Bloch and van den Nouweland (2020) and history-dependent expectations in Dutta and Vartianen (2020). 
is destroyed by a myopic player, then this player strictly prefers the resulting matching to the current matching and if a match is destroyed by a farsighted player, then this player strictly prefers the end matching to the current matching. If a match is added, then a myopic player involved must strictly prefer the resulting matching to the current matching and a farsighted player involved must strictly prefer the end matching to the current matching.

The notion of pairwise myopic-farsighted stable set has been introduced in Herings, Mauleon and Vannetelbosch (2020), but differs from the approach in this paper in one crucial aspect. In Herings, Mauleon and Vannetelbosch (2020) it is sufficient that one of the two players in a blocking pair makes a strict improvement, whereas here we require all players involved in an improving path to make strict improvements. This difference turns out to have major implications for the results to be obtained. Since both this paper and Herings, Mauleon and Vannetelbosch (2020) assume preferences to be strict, the changes in results are not caused by the classical problems in matching theory caused by indifferences in the preference relations.

Confirming the earlier literature, in homogeneous societies, where either all players are myopic or all players are farsighted, the core and the pairwise myopicfarsighted stable set provide the same predictions. Any core element is part of a pairwise myopic-farsighted stable set and elements outside the core are never part of a pairwise myopic-farsighted stable set.

For the heterogeneous societies, we first identify a condition that restores the equivalence between the core and the myopic-farsighted stable set. For matching problems satisfying the top-coalition property, the pairwise myopic-farsighted stable set is unique and equal to the core. This result holds independently from the distribution of myopic and farsighted players over the two sides of the marriage market.

Things become different when the top-coalition property is not satisfied. Consider marriage problems where all women are farsighted. The only core element that can be part of a pairwise myopic-farsighted stable set is the woman-optimal stable matching. This result shows the when myopic and farsighted players interact, not all core elements are equally relevant. Moreover, if all men are myopic, then the woman-optimal stable matching can only constitute a pairwise myopicfarsighted stable set if it is not Pareto dominated from the women point of view by an individually rational matching, i.e., if there does not exist an individually ratio- 
nal matching that is weakly preferred to the woman-optimal stable matching by all women and strictly preferred by some women. Otherwise, if the woman-optimal stable matching is Pareto dominated from the women point of view, then no pairwise myopic-farsighted stable set can be formed by core elements. Under these circumstances, the core and the pairwise myopic-farsighted stable set will always provide different predictions. This result shows that the core might not no longer be the relevant concept when myopic and farsighted players interact.

In the definition of the pairwise myopic-farsighted stable set, blocking has been restricted to pairs and single players. Standard notions of the vNM stable set allow for blocking by arbitrary coalitions and not only by coalitions of size one or two. We study whether general blocking is equivalent to pairwise blocking in marriage problems with both myopic and farsighted players. We find that there is an equivalence between the pairwise myopic-farsighted stable set and the coalitional myopic-farsighted stable set.

Herings, Mauleon and Vannetelbosch (2020) do not require that all players involved in a myopic-farsighted improving path make strict improvements. In that case, the woman-optimal stable matching always constitutes a pairwise myopicfarsighted stable set when all women are farsighted. Moreover, it can happen that other core elements, like the man-optimal stable matching, constitute a pairwise myopic-farsighted stable set. Finally, the robustness towards coalitional deviations gets lost.

The paper is organized as follows. Section 2 introduces marriage problems and standard notions of stability. Section 3 defines the pairwise myopic-farsighted stable set. It provides an example where all women are farsighted and all men are myopic and the unique pairwise myopic-farsighted stable set contains a matching outside the core. Section 4 presents the analysis on the relation between the core and the pairwise myopic-farsighted stable set. Section 5 investigates the equivalence between pairwise and coalitional blocking. Section 6 compares the results to those in Herings, Mauleon and Vannetelbosch (2020). Section 7 contains the conclusion.

\section{Marriage Problems}

A marriage problem consists of a finite set of players $N$, partitioned into a set of men $M$ and a set of women $W$. The set of non-empty subsets of $N$ is denoted by $\mathcal{N}$. 
Players can be either farsighted or myopic. The farsighted players are collected in the set $F \subset N$, which is allowed to be empty or equal to $N$. Myopic players only care about immediate payoffs, whereas farsighted players take a long-run perspective. The implications for behavior of being either myopic or farsighted, are made precise in Section 3.

Each player $i \in N$ has a complete and transitive preference ordering $\succ_{i}$ over the players of the opposite sex and the prospect of being alone. Preferences are assumed to be strict. We write $j \sim_{i} j^{\prime}$ if player $i$ is indifferent between $j$ and $j^{\prime}$, which can only be the case if $j=j^{\prime}$, and $j \succeq_{i} j^{\prime}$ if $j \succ_{i} j^{\prime}$ or $j \sim_{i} j^{\prime}$. Let $\succ=\left(\left(\succ_{m}\right)_{m \in M},\left(\succ_{w}\right)_{w \in W}\right)$ be a preference profile.

A marriage problem is a tuple $(M, W, F \succ)$. A matching is a function $\mu: N \rightarrow N$ satisfying the following properties:

(i) For every $m \in M, \mu(m) \in W \cup\{m\}$.

(ii) For every $w \in W, \mu(w) \in M \cup\{w\}$.

(iii) For every $i \in N, \mu(\mu(i))=i$.

The set of all matchings is denoted by $\mathcal{M}$. Given a matching $\mu \in \mathcal{M}$, player $i$ is said to be single if $\mu(i)=i$. A matching $\mu$ is individually rational if each player is acceptable to his or her partner, so for every $i \in N$ it holds that $\mu(i) \succeq_{i} i$. Let $\mathcal{I}$ be the set of matchings that are individually rational, i.e.,

$$
\mathcal{I}=\left\{\mu \in \mathcal{M} \mid \text { for every } i \in N, \mu(i) \succeq_{i} i\right\}
$$

A matching $\mu$ that is not individually rational can be blocked by a single player with an unacceptable partner. For a given matching $\mu$, a pair $\{m, w\}$ is said to form a blocking pair if $m$ and $w$ are not matched to one another but prefer each other to their partners at $\mu$, i.e. $w \succ_{m} \mu(m)$ and $m \succ_{w} \mu(w)$. A matching $\mu$ is stable if it is not blocked by any single player or any pair of players.

Given a matching $\mu \in \mathcal{M}$ with man $m \in M$ matched to woman $w \in W$, so $\mu(m)=w$, the matching $\mu^{\prime}$ that is identical to $\mu$, except that the match between $m$ and $w$ has been destroyed by either $m$ or $w$, is denoted by $\mu-(m, w)$. Given a matching $\mu \in \mathcal{M}$ such that $m \in M$ and $w \in W$ are not matched to one another, the matching $\mu^{\prime}$ that is identical to $\mu$, except that the pair $(m, w)$ has formed at 
$\mu^{\prime}$ and their partners at $\mu$, i.e., $\mu(w)$ and $\mu(m)$, are now single at $\mu^{\prime}$, is denoted by $\mu+(m, w)$.

For every $i \in N$, we extend the preference ordering $\succ_{i}$ over the player's potential partners to the set of matchings $\mathcal{M}$ in the following way. We say that player $i$ prefers the matching $\mu^{\prime}$ to the matching $\mu$ if $\mu^{\prime}(i) \succ_{i} \mu(i)$ and we write $\mu^{\prime} \succ_{i} \mu$. We write $\mu^{\prime} \sim_{i} \mu$ if $\mu^{\prime}(i) \sim_{i} \mu(i)$, and $\mu^{\prime} \succeq_{i} \mu$ if $\mu^{\prime} \succ_{i} \mu$ or $\mu^{\prime} \sim_{i} \mu$. For $S \in \mathcal{N}$, $\mu(S)=\{\mu(i) \mid i \in S\}$ denotes the set of partners of players in $S$ at $\mu$. A coalition $S \in \mathcal{N}$ is said to block a matching $\mu \in \mathcal{M}$ if there exists a matching $\mu^{\prime} \in \mathcal{M}$ such that $\mu^{\prime}(S)=S$ and $\mu^{\prime} \succ_{S} \mu$, where $\mu^{\prime} \succ_{S} \mu$ is defined as $\mu^{\prime}(i) \succ_{i} \mu(i)$ for every $i \in S$. The core of the marriage problem $(M, W, F, \succ)$ consists of all matchings that are not blocked by any coalition. We denote the set of matchings that belong to the core by $C$.

It has been shown by Gale and Shapley (1962) that the core of a marriage problem is non-empty. Also, a matching is stable if and only if it is not blocked by a coalition of size one or two if and only if it belongs to the core, see Theorem 3.3 in Roth and Sotomayor (1990). Knuth (1976) has shown that the core of a marriage problem is a distributive lattice. In particular, there is a man-optimal stable matching $\mu^{\mathrm{M}}$ and a woman-optimal stable matching $\mu^{\mathrm{W}}$. For any matching $\mu$ in the core, for every $m \in M$, it holds that $\mu^{\mathrm{M}} \succeq_{m} \mu$. Similarly, for any matching $\mu$ in the core, for every $w \in W$, it holds that $\mu^{\mathrm{W}} \succeq_{w} \mu$.

\section{The Pairwise Myopic-Farsighted Stable Set}

We propose the notion of the pairwise myopic-farsighted stable set to study the matchings that are stable when myopic and farsighted players interact with each other. This notion has been introduced in Herings, Mauleon and Vannetelbosch (2020) when deviating pairs are required to make weak improvements. Here we require strict improvements for all players involved in a deviation.

A pairwise myopic-farsighted improving path is a sequence of matchings that can emerge when farsighted players form or destroy matches based on the improvement the end matching offers them relative to the current one while myopic players form or destroy matches based on the improvement the next matching in the sequence offers them relative to the current one.

Definition 1. Let $(M, W, F, \succ)$ be a marriage problem. A pairwise myopic-farsighted 
improving path from a matching $\mu \in \mathcal{M}$ to a matching $\mu^{\prime} \in \mathcal{M} \backslash\{\mu\}$ is a finite sequence of distinct matchings $\mu_{0}, \ldots, \mu_{L}$ with $\mu_{0}=\mu$ and $\mu_{L}=\mu^{\prime}$ such that for every $\ell \in\{0, \ldots, L-1\}$ either (i) or (ii) holds:

(i) $\mu_{\ell+1}=\mu_{\ell}-(m, w)$ for some $(m, w) \in M \times W$ such that

$$
\begin{cases}\mu_{\ell+1}(m) \succ_{m} \mu_{\ell}(m) & \text { if } m \in M \backslash F \\ \mu_{L}(m) \succ_{m} \mu_{\ell}(m) & \text { if } m \in F\end{cases}
$$

or

$$
\begin{cases}\mu_{\ell+1}(w) \succ_{w} \mu_{\ell}(w) & \text { if } w \in W \backslash F \\ \mu_{L}(w) \succ_{w} \mu_{\ell}(w) & \text { if } w \in F .\end{cases}
$$

(ii) $\mu_{\ell+1}=\mu_{\ell}+(m, w)$ for some $(m, w) \in M \times W$ such that

$$
\begin{cases}\mu_{\ell+1}(m) \succ_{m} \mu_{\ell}(m) & \text { if } m \in M \backslash F, \\ \mu_{L}(m) \succ_{m} \mu_{\ell}(m) & \text { if } m \in F\end{cases}
$$

and

$$
\left\{\begin{array}{ll}
\mu_{\ell+1}(w) \succ_{w} \mu_{\ell}(w) & \text { if } w \in W \backslash F, \\
\mu_{L}(w) \succ_{w} \mu_{\ell}(w) & \text { if } w \in F
\end{array} .\right.
$$

Each matching in the sequence differs from the previous one in that either an existing match in the previous matching is destroyed like in case (i) or a new match is formed between a man and a woman that are not matched to one another in the previous matching as in case (ii).

The notion of a pairwise-myopic farsighted improving path differs from the one in Herings, Mauleon and Vannetelbosch (2020) in that all members involved in a coalition which is active on the path are required to improve strictly. Even under the assumption of strict preferences, as assumed in this paper, this makes a difference. In particular, when a farsighted player is currently involved in a match that is the same as in the end matching, then the farsighted player is not going to move when strict improvements for all players are required. On the contrary, if it is sufficient that at least one of the two players improves strictly, then such a farsighted player is willing to move together with another player who makes a strict improvement. If the other player is myopic, then the next matching in the sequence can be either better of worse for the farsighted player and will be changed at least once more to 
make the farsighted player indifferent with the end matching. If the other player is farsighted, then both players realize they will get separated from each other in the end matching, with the indifferent farsighted player returning to his original match.

If there exists a pairwise myopic-farsighted improving path from a matching $\mu$ to a matching $\mu^{\prime}$, then we write $\mu \rightarrow \mu^{\prime}$. The set of all matchings that can be reached from a matching $\mu \in \mathcal{M}$ by a pairwise myopic-farsighted improving path is denoted by $h^{*}(\mu)$, so

$$
h^{*}(\mu)=\left\{\mu^{\prime} \in \mathcal{M} \mid \mu \rightarrow \mu^{\prime}\right\} .
$$

Example 1. Consider the marriage problem $(M, W, F, \succ)$, which corresponds to Example 2.31 of Roth and Sotomayor (1990) with the roles of men and women reversed. It holds that $M=\left\{m_{1}, m_{2}, m_{3}\right\}$ and $W=\left\{w_{1}, w_{2}, w_{3}\right\}$. Assume $F=$ $\left\{w_{1}, w_{2}, w_{3}\right\}$, so all women are farsighted and all men are myopic. The preferences of the players are as follows.

$\begin{array}{lllllll}\frac{m_{1}}{w_{1}} & \frac{m_{2}}{w_{3}} & \frac{m_{3}}{w_{1}} & & \frac{w_{1}}{m_{2}} & \frac{w_{2}}{m_{1}} & \frac{w_{3}}{m_{1}} \\ w_{2} & w_{1} & w_{2} & m_{1} & m_{2} & m_{2} \\ w_{3} & w_{2} & w_{3} & & m_{3} & m_{3} & m_{3} .\end{array}$

By applying the deferred acceptance algorithm of Gale and Shapley (1962), it can be easily verified that the woman-optimal stable matching is equal to

$$
\begin{aligned}
& \mu^{\mathrm{W}}\left(m_{1}\right)=w_{1}, \\
& \mu^{\mathrm{W}}\left(m_{2}\right)=w_{3}, \\
& \mu^{\mathrm{W}}\left(m_{3}\right)=w_{2} .
\end{aligned}
$$

The matching $\bar{\mu}$ defined by

$$
\begin{aligned}
& \bar{\mu}\left(m_{1}\right)=w_{3}, \\
& \bar{\mu}\left(m_{2}\right)=w_{1}, \\
& \bar{\mu}\left(m_{3}\right)=w_{2},
\end{aligned}
$$

is strictly preferred by $w_{1}$ and $w_{3}$ to $\mu^{\mathrm{W}}$ and does not make a difference for $w_{2}$. However, the pair $\left(m_{1}, w_{2}\right)$ can block $\bar{\mu}$, so $\bar{\mu}$ does not belong to the core.

A matching $\bar{\mu} \in \mathcal{M}$ is said to $W$-dominate a matching $\mu \in \mathcal{M}$ if $\bar{\mu}(i) \succeq_{i} \mu(i)$ for all $i \in W$ and $\bar{\mu}(i) \succ_{i} \mu(i)$ for some $i \in W$. In this case, we also say that the matching $\bar{\mu}$ Pareto dominates $\mu$ from the woman point of view. We show that 
$\bar{\mu} \in h^{*}\left(\mu^{\mathrm{W}}\right)$, so it is possible that farsighted women leave the woman-optimal stable matching to end up in a matching that $W$-dominates $\mu^{\mathrm{W}}$. To see this, consider the pairwise myopic-farsighted improving path $\mu_{0}, \ldots, \mu_{4}$ with $\mu_{0}=\mu^{\mathrm{W}}$ and $\mu_{4}=\bar{\mu}$, where $\mu_{1}=\mu_{0}-\left(m_{1}, w_{1}\right), \mu_{2}=\mu_{1}-\left(m_{2}, w_{3}\right), \mu_{3}=\mu_{2}+\left(m_{2}, w_{1}\right)$, and $\mu_{4}=$ $\mu_{3}+\left(m_{1}, w_{3}\right)$. This pairwise myopic-farsighted improving path is illustrated in Figure 1.

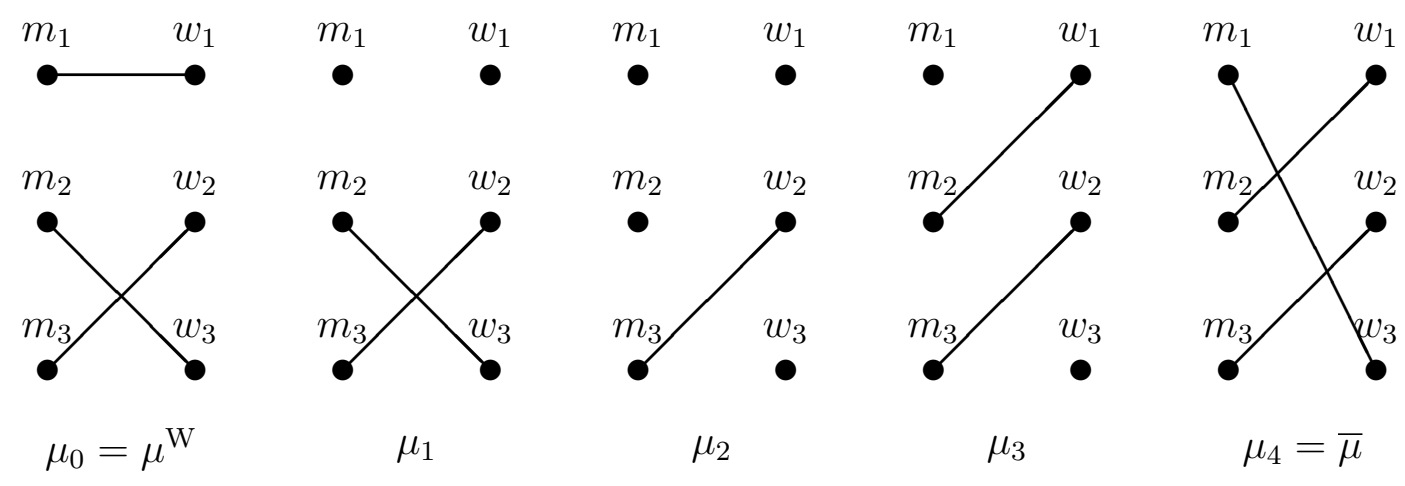

Figure 1: Pairwise myopic-farsighted improving path in Example 1 to move from $\mu^{\mathrm{W}}$ to $\bar{\mu}$.

The move to $\mu_{1}$ is initiated by $w_{1}$ who is farsighted and therefore wants to destroy her match with $m_{1}$ in the anticipation of ending up in a match with $m_{2}$. Similarly, the move from $\mu_{1}$ to $\mu_{2}$ is initiated by $w_{3}$ who is farsighted and is willing to divorce $m_{2}$ in the expectation of being matched with $m_{1}$. The transition to $\mu_{4}=\bar{\mu}$ is completed by the subsequent marriages of the single players $m_{2}$ and $w_{1}$ and the single players $m_{1}$ and $w_{3}$.

At the same time, it holds that $\mu^{\mathrm{W}} \notin h^{*}(\bar{\mu})$. Indeed, both $w^{1}$ and $w^{3}$ are strictly worse off at $\mu^{W}$ and $w^{2}$ is indifferent, so none of the women is willing to add or destroy a match at $\bar{\mu}$ in the anticipation of ending up at $\mu^{\mathrm{W}}$. Since all the men are myopic and have an individually rational match at $\bar{\mu}$, they are not willing to destroy a link, so it is not possible to find a pairwise myopic-farsighted improving path from $\bar{\mu}$ to $\mu^{\mathrm{W}}$. It holds that $\mu^{\mathrm{W}} \notin h^{*}(\bar{\mu})$.

The pairwise myopic-farsighted stable set results when the conditions of internal and external stability are based on the pairwise myopic-farsighted improving paths. 
Definition 2. Let $(M, W, F, \succ)$ be a marriage problem. A set of matchings $V \subset \mathcal{M}$ is a pairwise myopic-farsighted stable set if it satisfies:

(i) Internal stability: For every $\mu, \mu^{\prime} \in V$, it holds that $\mu^{\prime} \notin h^{*}(\mu)$.

(ii) External stability: For every $\mu \in \mathcal{M} \backslash V$, it holds that $h^{*}(\mu) \cap V \neq \emptyset$.

Condition (i) of Definition 2 corresponds to internal stability. For any two matchings $\mu$ and $\mu^{\prime}$ in the pairwise myopic-farsighted stable set $V$ it does not hold that $\mu \rightarrow \mu^{\prime}$. Condition (ii) of Definition 2 expresses external stability. For every matching $\mu$ outside the pairwise myopic-farsighted stable set $V$ it holds that there is $\mu^{\prime} \in V$ such that $\mu \rightarrow \mu^{\prime}$.

Recall that in Example 1, the non-core element $\bar{\mu}$ is strictly preferred by two out of three women to $\mu^{\mathrm{W}}$, whereas the third woman is indifferent. The next example shows that $\{\bar{\mu}\}$ is a pairwise myopic-farsighted stable set. This example is important since it shows that in heterogeneous societies, it is possible for the farsighted side to do better than in any core element.

Example 2. We take the same primitives as in Example 1. In Example 1 it has been shown that $\bar{\mu} \in h^{*}\left(\mu^{\mathrm{W}}\right)$. We show now that for every $\mu \in \mathcal{M} \backslash\{\bar{\mu}\}$ it holds that $\bar{\mu} \in h^{*}(\mu)$. It then follows that $V=\{\bar{\mu}\}$ is a pairwise myopic-farsighted stable set.

Let some $\mu \in \mathcal{M} \backslash\{\bar{\mu}\}$ be given. We distinguish four cases.

Case 1. $\mu\left(m_{1}\right)=w_{2}$ and $\mu\left(m_{2}\right)=w_{1}$.

It follows that $\mu\left(m_{3}\right)=m_{3}$ or $\mu\left(m_{3}\right)=w_{3}$. We define $\mu_{0}=\mu$ and $\mu_{1}=\mu_{0}+\left(m_{2}, w_{3}\right)$. Notice that $\bar{\mu}\left(w_{3}\right)=m_{1} \succ_{w_{3}} \mu_{0}\left(w_{3}\right)$ and $w_{3} \succ_{m_{2}} \mu_{0}\left(m_{2}\right)$, so $m_{2}$ and $w_{3}$ are willing to form the match and reach $\mu_{1}$. Next, we take $\mu_{2}=\mu_{1}+\left(m_{1}, w_{1}\right)$. The purpose of this step is to make $w_{2}$ single. Woman $w_{1}$ is strictly improving since $\bar{\mu}\left(w_{1}\right)=m_{2} \succ_{w_{1}}$ $w_{1}=\mu_{1}\left(w_{1}\right)$. Man $m_{1}$ is willing to match $w_{1}$ since $w_{1} \succ_{m_{1}} \mu_{1}\left(m_{1}\right)=w_{2}$. Next, we make $w_{1}$ and $w_{3}$ single by setting $\mu_{3}=\mu_{2}-\left(m_{1}, w_{1}\right)$ and $\mu_{4}=\mu_{3}-\left(m_{2}, w_{3}\right)$. Woman $w_{1}$ is willing to destroy the match at $\mu_{2}$ since $\bar{\mu}\left(w_{1}\right)=m_{2} \succ_{w_{1}} m_{1}$ and $w_{3}$ is willing to destroy the match at $\mu_{3}$ since $\bar{\mu}\left(w_{3}\right)=m_{1} \succ_{w_{3}} m_{2}$. Notice that at $\mu_{4}$, everyone is single. We now define $\mu_{5}=\mu_{4}+\left(m_{1}, w_{3}\right), \mu_{6}=\mu_{5}+\left(m_{2}, w_{1}\right)$, and $\mu_{7}=\mu_{6}+\left(m_{3}, w_{2}\right)$. It is easily verified that the latter three moves involve strict improvements for the marrying couple. Since $\mu_{7}=\bar{\mu}$, we have shown that $\mu_{0}, \ldots, \mu_{7}$ is a pairwise myopic-farsighted improving path from $\mu$ to $\bar{\mu}$. 
Case 2. $\mu\left(m_{1}\right)=w_{2}$ and $\mu\left(m_{2}\right) \neq w_{1}$.

We define $\mu_{0}=\mu$ and $\mu_{1}=\mu_{0}+\left(m_{1}, w_{1}\right)$. Notice that $\bar{\mu}\left(w_{1}\right)=m_{2} \succ_{w_{1}} \mu_{0}\left(w_{1}\right)$, with $\mu_{0}\left(w_{1}\right)=m_{3}$ or $\mu_{0}\left(w_{1}\right)=w_{1}$, and $w_{1} \succ_{m_{1}} \mu_{0}\left(m_{1}\right)$, so $m_{1}$ and $w_{1}$ are willing to form the match and reach $\mu_{1}$. Woman $w_{2}$ is now single. At $\mu_{1}$, if $w_{3}$ is married, she is willing to destroy the match at $\mu_{1}$, since $\bar{\mu}\left(w_{3}\right)=m_{1} \succ_{w_{3}} m_{2}, m_{3}$, moving to $\mu_{2}=\mu_{1}-\left(\mu_{1}\left(w_{3}\right), w_{3}\right)$. Next, we also make $w_{1}$ single by setting $\mu_{3}=\mu_{2}-\left(m_{1}, w_{1}\right)$. Woman $w_{1}$ is willing to destroy the match at $\mu_{2}$ because $\bar{\mu}\left(w_{1}\right)=m_{2} \succ_{w_{1}} m_{1}$. Notice that at $\mu_{3}$, everyone is single. We now define $\mu_{4}=\mu_{3}+\left(m_{1}, w_{3}\right), \mu_{5}=\mu_{4}+\left(m_{2}, w_{1}\right)$, and $\mu_{6}=\mu_{5}+\left(m_{3}, w_{2}\right)$. It is easily verified that the latter three moves involve strict improvements for the marrying couple. Since $\mu_{6}=\bar{\mu}$, we have shown that $\mu_{0}, \ldots, \mu_{6}$ is a pairwise myopic-farsighted improving path from $\mu$ to $\bar{\mu}$.

Case 3. $\mu\left(m_{2}\right)=w_{2}$.

We define $\mu_{0}=\mu$ and $\mu_{1}=\mu_{0}+\left(m_{2}, w_{1}\right)$. The purpose of this step is to make $w_{2}$ single. Notice that $\bar{\mu}\left(w_{1}\right)=m_{2} \succ_{w_{1}} \mu_{0}\left(w_{1}\right)$ and $w_{1} \succ_{m_{2}} \mu_{0}\left(m_{2}\right)$, so $m_{2}$ and $w_{1}$ are willing to form the match and reach $\mu_{1}$. Next, we take $\mu_{2}=\mu_{1}+\left(m_{3}, w_{2}\right)$. Woman $w_{2}$ is willing to do so since $\bar{\mu}\left(w_{2}\right)=m_{3} \succ_{w_{2}} w_{2}=\mu_{1}\left(w_{2}\right)$. Man $m_{3}$ is willing to match $w_{2}$ since $w_{2} \succ_{m_{3}} \mu_{1}\left(m_{3}\right)$, with $\mu_{1}\left(m_{3}\right)=w_{3}$ or $\mu_{1}\left(m_{3}\right)=m_{3}$. Now, either $\mu_{2}=\bar{\mu}$ or we form the remaining match between $m_{1}$ and $w_{3}$ moving to $\mu_{3}=\mu_{2}+\left(m_{1}, w_{3}\right)=\bar{\mu}$.

Case 4. $\mu\left(m_{3}\right)=w_{2}$ or $\mu\left(w_{2}\right)=w_{2}$.

We define $\mu_{0}=\mu$. At $\mu_{0}$, if $w_{1}$ or $w_{3}$ are not married to their final partner at $\bar{\mu}$, then we proceed to divorce them moving to $\mu_{1}=\mu_{0}-\left(\mu_{0}\left(w_{1}\right), w_{1}\right)$ and then to $\mu_{2}=\mu_{1}-\left(\mu_{1}\left(w_{3}\right), w_{3}\right)$. Next, we form the remaining matches at $\bar{\mu}$ one by one.

We show next that $\{\bar{\mu}\}$ is the unique pairwise myopic-farsighted stable set in Example 2.

Example 3. We take the same primitives as in Example 2. It follows from the analysis in Example 2 that for every $\mu \in \mathcal{M} \backslash\{\bar{\mu}\}$ it holds that $\bar{\mu} \in h^{*}(\mu)$. It then follows that $V=\{\bar{\mu}\}$ is a pairwise myopic-farsighted stable set.

Suppose now that $V$ is a pairwise myopic-farsighted stable set that does not contain $\bar{\mu}$. By external stability of $V$, we know that $V$ must contain some matching in $h^{*}(\bar{\mu})$. It can be verified that $h^{*}(\bar{\mu})=\left\{\mu^{1}, \mu^{2}, \mu^{3}, \mu^{4}\right\}$, where $\mu^{1}=\bar{\mu}+\left(m_{1}, w_{2}\right)$, $\mu^{2}=\mu^{1}+\left(m_{3}, w_{3}\right), \mu^{3}=\mu^{1}+\left(m_{2}, w_{3}\right)$ and $\mu^{4}=\mu^{3}+\left(m_{3}, w_{1}\right)$. The move from $\bar{\mu}$ to $\mu^{1}$ is done by the pair $\left(m_{1}, w_{2}\right)$ with both $m_{1}$ and $w_{2}$ strictly preferring $\mu^{1}$ to $\bar{\mu}$. Next, the move from $\mu^{1}$ to $\mu^{2}$ is realized by the pair $\left(m_{3}, w_{3}\right)$ with both $m_{3}$ and $w_{3}$ 
strictly preferring $\mu^{2}$ to $\mu^{1}$. Notice that $w_{2}$ is strictly better off at $\mu^{2}$ compared to $\bar{\mu}$. The move from $\mu^{1}$ to $\mu^{3}$ is realized by the pair $\left(m_{2}, w_{3}\right)$ with both $m_{2}$ and $w_{3}$ strictly preferring $\mu^{3}$ to $\mu^{1}$. Notice that $w_{2}$ is strictly better off at $\mu^{3}$ compared to $\bar{\mu}$. And, finally, $\mu^{4}$ is obtained from $\mu^{3}$ by forming the pair $\left(m_{3}, w_{1}\right)$ with both $m_{3}$ and $w_{1}$ strictly preferring $\mu^{4}$ to $\mu^{3}$. Notice that $w_{2}$ is strictly better off at $\mu^{4}$ compared to $\bar{\mu}$ and that $w_{3}$ is also strictly better off at $\mu^{4}$ compared to $\mu^{1}$. Thus, we know that $\left\{\mu^{1}, \mu^{2}, \mu^{3}, \mu^{4}\right\} \cap V \neq \emptyset$.

We argue next that $\mu^{\mathrm{W}} \in h^{*}\left(\mu^{1}\right), \mu^{\mathrm{W}} \in h^{*}\left(\mu^{2}\right), \mu^{\mathrm{W}} \in h^{*}\left(\mu^{3}\right)$, and $\mu^{\mathrm{W}} \in h^{*}\left(\mu^{4}\right)$. Indeed, we have that $\mu^{1}+\left(m_{2}, w_{3}\right)+\left(m_{1}, w_{1}\right)+\left(m_{3}, w_{2}\right)=\mu^{\mathrm{W}}$ and $\mu^{2}+\left(m_{2}, w_{3}\right)+$ $\left(m_{1}, w_{1}\right)+\left(m_{3}, w_{2}\right)=\mu^{\mathrm{W}}$. The men are myopically improving in each step and the women all prefer $\mu^{\mathrm{W}}$ to the matching before moving. Also, we have that $\mu^{3}+\left(m_{1}, w_{1}\right)+\left(m_{3}, w_{2}\right)=\mu^{\mathrm{W}}$ and $\mu^{4}+\left(m_{1}, w_{1}\right)+\left(m_{3}, w_{2}\right)=\mu^{\mathrm{W}}$. The men are myopically improving in each step and the women all prefer $\mu^{\mathrm{W}}$ to the matching before moving. Thus, by internal stability of $V$, we have that $\mu^{\mathrm{W}} \notin V$. It can be verified that $h^{*}\left(\mu^{\mathrm{W}}\right)=\{\bar{\mu}\}$. We therefore have that $h^{*}\left(\mu^{\mathrm{W}}\right) \cap V=\emptyset$, so $V$ violates external stability, a contradiction. Consequently, there is a unique pairwise myopicfarsighted stable set $V=\{\bar{\mu}\}$.

Example 3 shows that the unique pairwise myopic-farsighted may not contain a core element. Hence, Example 3 shows that, in the presence of myopic and farsighted players, it is possible that the core and the pairwise myopic-farsighted stable set provide different predictions.

\section{Pairwise Myopic-Farsighted Stable Sets and the Core}

In this section we investigate the relation between the pairwise myopic-farsighted stable set and the core.

In the special case where all players are myopic, $F=\emptyset$, the pairwise myopicfarsighted stable set is equivalent to the pairwise CP vNM set of Herings, Mauleon and Vannetelbosch (2017) and is equal to the core.

Mauleon, Vannetelbosch and Vergote (2011) show that the vNM farsightedly stable sets are characterized as the singletons consisting of a core element. In Section 5 
we show that the results in case coalitions of arbitrary size are allowed to move coincide with those when moves are made by singletons and pairs. The pairwise myopic-farsighted stable sets are therefore characterized as singleton core elements as well in case $F=N$, so the special case where all players are farsighted.

We now turn to the heterogeneous case. One popular restriction that guarantees uniqueness of the core is the top-coalition property, introduced by Banerjee, Konishi and Sönmez (2001).

Definition 3. Let $(M, W, F, \succ)$ be a marriage problem. Let $S \in \mathcal{N}$. A non-empty coalition $T \subset S$ is a top-coalition of $S$ if every $i \in T$ is matched to the most preferred partner in $S$. The marriage problem $(M, W, F, \succ)$ satisfies the top-coalition property if every coalition $S \in \mathcal{N}$ contains a top coalition of $S$.

Definition 3 implies that for any group of players there are always two players that top rank each other or there is a player who prefers to remain single. For $k=1, \ldots, \ell$, let $S_{k}^{*}$ be a top coalition of $N \backslash \cup_{j=1}^{k-1} S_{j}^{*}$ involving one or two players and let $\cup_{k=1}^{\ell} S_{k}^{*}=N$. The matching $\mu^{*}$ such that for every $i \in N$ there is $k \in$ $\{1, \ldots, \ell\}$ such that $S_{k}^{*}=\left\{i, \mu^{*}(i)\right\}$ is called the matching induced by the partition $\left\{S_{1}^{*}, S_{2}^{*}, \ldots, S_{k}^{*}\right\}$. Banerjee, Konishi and Sönmez (2001) have shown that when the top-coalition property is satisfied, the core is unique and consists of the matching $\mu^{*}$. It follows that the partition $\left\{S_{1}^{*}, S_{2}^{*}, \ldots, S_{k}^{*}\right\}$, called the top-coalition partition, is unique as well.

We show next that for matching problems satisfying the top-coalition property, the pairwise myopic-farsighted stable set is unique and equal to the core.

Theorem 1. Let $(M, W, F, \succ)$ be a marriage problem satisfying the top-coalition property. Then $V$ is a pairwise myopic-farsighted stable set if and only if $V=C$.

Whenever the top-coalition property is satisfied, the core and the pairwise myopicfarsighted stable set make exactly the same predictions. An interesting aspect of this result is that the distribution of myopic and farsighted players over the two sides of the market does not matter. Consider the case of a single farsighted player together with only myopic players. Assume that at some moment in the matching process, the farsighted player can form a match with another player which makes the farsighted player strictly better off than in the unique core element. There is no hope for the farsighted player that such an improvement would be long-lasting. 
We now turn to the case where all women are farsighted, whereas any given man can be either myopic or farsighted, i.e., $W \subset F$. It is convenient to define the set of women that strictly prefer a matching $\mu \in \mathcal{M}$ to $\mu^{\mathrm{W}}$ by $W(\mu)$, so

$$
W(\mu)=\left\{w \in W \mid \mu(w) \succ_{w} \mu^{\mathrm{W}}(w)\right\}
$$

It follows from the lattice structure of the core that if $\mu \in C$, then we have $W(\mu)=\emptyset$.

A matching $\mu \in \mathcal{M}$ is said to $W$-dominate the matching $\mu^{\mathrm{W}}$ if, for every $w \in$ $W, \mu(w) \succeq_{w} \mu^{\mathrm{W}}(w)$ and $W(\mu) \neq \emptyset$. The next result states that if $\mu^{\mathrm{W}}$ is not $W$ dominated by an individually rational matching, then $\left\{\mu^{\mathrm{W}}\right\}$ is a pairwise myopicfarsighted stable set.

Theorem 2. Let $(M, W, F, \succ)$ be a marriage problem with $F \supset W$. Assume that $\mu^{\mathrm{W}}$ is not $W$-dominated by a matching in $\mathcal{I}$. Then $\left\{\mu^{\mathrm{W}}\right\}$ is a pairwise myopic-farsighted stable set.

Proof. Since $\left\{\mu^{\mathrm{W}}\right\}$ is a singleton, Condition (i) of Definition 2, internal stability, is satisfied. Condition (ii) of Definition 2, external stability, follows from Lemmas 1 and 2 that are proved in the appendix.

Theorems 1 and 2 have some overlap. If a matching problem satisfies the topcoalition property, then $\mu^{\mathrm{W}}$ cannot be $W$-dominated by a matching in $\mathcal{I}$, so in case $F \supset W$, the result of Theorem 1 follows from Theorem 2 as well. Theorem 1 does not depend on the assumption that $F \supset W$. Theorem 2 makes a weaker assumption on the preferences, but a stronger assumption on the set of farsighted players.

The proof that $\mu^{\mathrm{W}}$ satisfies external stability relies on Lemmas 1 and 2. We prove in Lemma 1 that the woman-optimal stable matching $\mu^{\mathrm{W}}$ can be reached from any matching $\mu$ different from $\mu^{\mathrm{W}}$ with the property that, for every $w \in W$, $\mu^{\mathrm{W}}(w) \succeq_{w} \mu(w)$. This also covers the case where $\mu$ is a core element different from $\mu^{\mathrm{W}}$. The proof of Lemma 1 first identifies the set $W^{1}$ of women that strictly prefer $\mu^{\mathrm{W}}$ to $\mu$ and that are not single at $\mu$. Since all women are farsighted, they are willing to divorce their men. The resulting matching is such that all women are either married to their partner at $\mu^{\mathrm{W}}$ or are single. The latter women now marry their partner at $\mu^{\mathrm{W}}$.

Lemma 2 considers the case where the matching $\mu$ is such that some women prefer $\mu$ to $\mu^{\mathrm{W}}$, so the set of women $W(\mu)$ that strictly prefer their match at $\mu$ to the one at $\mu^{\mathrm{W}}$ is non-empty. We prove in Lemma 2 that, if $\mu^{\mathrm{W}}$ is not $W$-dominated 
by a matching in $\mathcal{I}$, then the woman-optimal stable matching $\mu^{\mathrm{W}}$ can be reached from any matching $\mu$ different from $\mu^{\mathrm{W}}$ such that $W(\mu) \neq \emptyset$.

The proof of Lemma 2 proceeds as follows. In order to reach $\mu^{\mathrm{W}}$ departing from $\mu$, first the matches that are not individually rational at $\mu$ are destroyed until we arrive at an individually rational matching $\mu_{k^{1}}$. We then identify all the women that prefer $\mu_{k^{1}}$ to $\mu^{\mathrm{W}}$ and are married to a farsighted man. Since $\mu^{\mathrm{W}}$ is a core element, it follows that such a farsighted man prefers $\mu^{\mathrm{W}}$ to $\mu_{k^{1}}$ and is willing to destroy the match with his partner. In this way we obtain a matching $\mu_{k^{1}+k^{2}}$ such that all women that prefer $\mu_{k^{1}+k^{2}}$ to $\mu^{\mathrm{W}}$ are married to a myopic man. If $W\left(\mu_{k^{1}+k^{2}}\right)=\emptyset$, then we can proceed as in the proof of Lemma 1. Consider the case that $W\left(\mu_{k^{1}+k^{2}}\right) \neq \emptyset$. The set of men married to $W\left(\mu_{k^{1}+k^{2}}\right)$ at $\mu_{k^{1}+k^{2}}$ cannot be the same as the set of men married to $W\left(\mu_{k^{1}+k^{2}}\right)$ at $\mu^{\mathrm{W}}$, since otherwise we can construct an individually rational matching that $W$-dominates $\mu^{\mathrm{W}}$, which violates the assumptions of Theorem 2. Since all women in $W\left(\mu_{k^{1}+k^{2}}\right)$ are married, we can select a man $m_{0}$ married to a woman in $W\left(\mu_{k^{1}+k^{2}}\right)$ at $\mu_{k^{1}+k^{2}}$, but is not married to a woman in $W\left(\mu_{k^{1}+k^{2}}\right)$ at $\mu^{\mathrm{W}}$. We show that the pair $\left(m_{0}, \mu^{\mathrm{W}}\left(m_{0}\right)\right)$ can block $\mu_{k^{1}+k^{2}}$. More generally, we generate a sequence of blocking pairs $\left(m_{\ell}, w_{\ell}\right)$ that blocks $\mu_{k^{1}+k^{2}+\ell}$ and such that $m_{\ell}=\mu^{\mathrm{W}}\left(w_{\ell}\right)$. We show that after a finite number of steps $k^{3}$ equal to the cardinality of $W\left(\mu_{k^{1}+k^{2}}\right)$, we arrive at a matching $\mu_{k^{1}+k^{2}+k^{3}}$ such that no woman strictly prefers $\mu_{k^{1}+k^{2}+k^{3}}$ to $\mu^{\mathrm{W}}$; i.e., $W\left(\mu_{k^{1}+k^{2}+k^{3}}\right)=\emptyset$. Thus, the matching $\mu_{k^{1}+k^{2}+k^{3}}$ either coincides with $\mu^{\mathrm{W}}$ or satisfies the assumptions of Lemma 1. In the latter case, we complete the pairwise myopic-farsighted improving path leading to $\mu^{\mathrm{W}}$ as in the proof of Lemma 1.

From Lemmas 1 and 2, it follows easily that $\left\{\mu^{\mathrm{W}}\right\}$ is a pairwise myopic-farsighted stable set.

We have shown that in case all women are farsighted and $\mu^{\mathrm{W}}$ is not $W$-dominated by a matching in $\mathcal{I}$, they can achieve the woman-optimal stable matching $\mu^{\mathrm{W}}$, irrespective of whether men are myopic or farsighted.

It follows from the next result that if all women are farsighted and all men are myopic, then no core element different from $\mu^{\mathrm{W}}$ can be a pairwise myopic-farsighted stable set.

Theorem 3. Let $(M, W, F, \succ)$ be a marriage problem with $F=W$. If $V \subset C$ is a pairwise myopic-farsighted stable set, then $V=\left\{\mu^{\mathrm{W}}\right\}$.

The proof of Theorem 3 argues that by internal stability, $V \subset C$ cannot contain 
$\mu^{\mathrm{W}}$ together with another core element, so the only possibility besides $\left\{\mu^{\mathrm{W}}\right\}$ for $V \subset C$ to be a pairwise myopic-farsighted stable set is that $V \subset C \backslash\left\{\mu^{\mathrm{W}}\right\}$. By external stability, there should now be a myopic-farsighted improving path from $\mu^{\mathrm{W}}$ to a core element. But with all women being farsighted, this is impossible.

When the women are farsighted and the men are myopic, the only possible pairwise myopic-farsighted stable set contained in the core consists of the womanoptimal stable matching. Theorem 2 presents a sufficient condition such that $\mu^{\mathrm{W}}$ is indeed a pairwise myopic-farsighted stable set. Theorem 4 shows that this sufficient condition is also necessary. This result generalizes Example 3 by showing that if there exists an individually rational matching that $W$-dominates $\mu^{\mathrm{W}}$, then no subset of the core can be a pairwise myopic-farsighted stable set.

Theorem 4. Let $(M, W, F, \succ)$ be a marriage problem with $F=W$. If $\mu^{\mathrm{W}}$ is $W$ dominated by a matching in $\mathcal{I}$, then a pairwise myopic-farsighted stable set cannot be contained in $C$.

The proof of Theorem 4 starts by observing that because of Theorem $3,\left\{\mu^{\mathrm{W}}\right\}$ is the only candidate for a pairwise myopic-farsighted stable set contained in $C$. But $\left\{\mu^{\mathrm{W}}\right\}$ cannot satisfy external stability, since farsighted women and myopic men will not reach $\mu^{\mathrm{W}}$ by a myopic-farsighted improving path when starting from an individually rational matching that $W$-dominates $\mu^{\mathrm{W}}$.

When all the women are farsighted and all the men are myopic and the womanoptimal stable matching is $W$-dominated by an individually rational matching, then no pairwise myopic-farsighted stable set can be formed by core elements. When this is the case, the core and the pairwise myopic-farsighted stable set will always provide different predictions. It follows that unlike in the homogeneous cases studied by the literature so far, the core might not be the relevant concept when myopic and farsighted players interact.

\section{Robustness to Coalitional Deviations}

Up to now, in the definition of the pairwise myopic-farsighted stable set, blocking has been restricted to be by pairs and single players. Standard notions of the vNM stable set allow for blocking by arbitrary coalitions and not only by coalitions of size one or two. In marriage problems, general blocking is equivalent to pairwise 
blocking and the core coincides with the set of stable matchings. In this section, we study whether general blocking is equivalent to pairwise blocking in marriage problems with both myopic and farsighted players.

Definition 4. Given a matching $\mu \in \mathcal{M}$, a coalition $S \in \mathcal{N}$ is able to enforce the matching $\mu^{\prime}$ if the following conditions hold: (i) $\mu^{\prime}(i) \notin\{\mu(i), i\}$ implies $\left\{i, \mu^{\prime}(i)\right\} \subset$ $S$ and (ii) $\mu^{\prime}(i)=i \neq \mu(i)$ implies $\{i, \mu(i)\} \cap S \neq \emptyset$.

If, given some matching $\mu$, coalition $S$ can enforce matching $\mu^{\prime}$, then any match in $\mu^{\prime}$ that does not exist in $\mu$ should be between players in $S$. Moreover, if a match in $\mu$ is destroyed, then one of the two players involved in that match should belong to $S$.

Definition 5. Let $(M, W, F, \succ)$ be a marriage problem. A coalitional myopicfarsighted improving path from a matching $\mu \in \mathcal{M}$ to a matching $\mu^{\prime} \in \mathcal{M} \backslash\{\mu\}$ is a finite sequence of distinct matchings $\mu_{0}, \ldots, \mu_{L}$ with $\mu_{0}=\mu$ and $\mu_{L}=\mu^{\prime}$ such that for every $\ell \in\{0, \ldots, L-1\}$ there is a coalition $S_{\ell} \in \mathcal{N}$ that can enforce $\mu_{\ell+1}$ from $\mu_{\ell}$ and

$$
\begin{array}{ll}
\mu_{\ell+1}(i) \succ_{i} \mu_{\ell}(i) & \text { if } i \in S_{\ell} \backslash F, \\
\mu_{L}(i) \succ_{i} \mu_{\ell}(i) & \text { if } i \in S_{\ell} \cap F .
\end{array}
$$

Let some $\mu \in \mathcal{M}$ be given. The set of matchings $\mu^{\prime} \in \mathcal{M}$ such that there is a coalitional myopic-farsighted improving path from $\mu$ to $\mu^{\prime}$ is denoted by $g^{*}(\mu)$. It clearly holds that $h^{*}(\mu) \subset g^{*}(\mu)$.

Definition 6. Let $(M, W, F, \succ)$ be a marriage problem. A set of matchings $V \subset \mathcal{M}$ is a coalitional myopic-farsighted stable set if it satisfies:

(i) Internal stability: For every $\mu, \mu^{\prime} \in V$, it holds that $\mu^{\prime} \notin g^{*}(\mu)$.

(ii) External stability: For every $\mu \in \mathcal{M} \backslash V$, it holds that $g^{*}(\mu) \cap V \neq \emptyset$.

The notion of the coalitional myopic-farsighted stable set in Definition 6 coincides with the definition of the vNM farsightedly stable set of Mauleon, Vannetelbosch and Vergote (2011) when all players are farsighted. Notice that pairwise myopicfarsighted stable sets and coalitional myopic-farsighted stable sets would coincide if $g^{*}$ and $h^{*}$ would coincide. It has already been observed in Herings, Mauleon, and 
Vannetelbosch (2017) that this is not the case if all players are myopic. Example 4 demonstrates that the same example can be used to show that $g^{*}$ and $h^{*}$ do not generally coincide irrespective of whether players are myopic or farsighted.

Example 4. Consider a matching problem $(M, W, F, \succ)$, where $M=\left\{m_{1}, m_{2}\right\}$, $W=\left\{w_{1}, w_{2}\right\}$, the set $F$ is taken arbitrary, and the preferences of the individuals are as follows.

$\begin{array}{lllll}\frac{m_{1}}{w_{1}} & \frac{m_{2}}{w_{2}} & & \frac{w_{1}}{w_{1}} & \frac{w_{2}}{w_{2}} \\ w_{2} & w_{1} & & m_{1} & m_{2} \\ m_{1} & m_{2} & & m_{2} & m_{1}\end{array}$

The preferences in Example 4 are such that man $m_{1}$ prefers to be married to $w_{1}$ rather than to $w_{2}$ and $m_{2}$ prefers to be married to $w_{2}$ rather than to $w_{1}$. Also woman $w_{1}$ prefers $m_{1}$ over $m_{2}$ and woman $w_{2}$ prefers $m_{2}$ over $m_{1}$, but contrary to the men, women prefer not to be married at all. The matching $\mu^{\prime}$ is defined by $\mu^{\prime}\left(m_{1}\right)=w_{2}$ and $\mu^{\prime}\left(m_{2}\right)=w_{1}$. The matching $\mu^{*}$ is defined by $\mu^{*}\left(m_{1}\right)=w_{1}$ and $\mu^{*}\left(m_{2}\right)=w_{2}$.

It is immediate that $\mu^{*} \in g^{*}\left(\mu^{\prime}\right)$, since the coalition $N$ can go from $\mu^{\prime}$ to $\mu^{*}$ in one step, leading to a strict improvement for every player.

We argue next that $\mu^{*} \notin h^{*}\left(\mu^{\prime}\right)$. The first observation is that at any $\mu \in h^{*}\left(\mu^{\prime}\right)$ it holds that at least one of the women is single. Suppose $\mu_{0}, \ldots, \mu_{L}$ is a pairwise myopic-farsighted improving path from $\mu_{0}=\mu^{\prime}$ to $\mu_{L}=\mu^{*}$. At $\mu_{1}$, exactly one of the women is single. Irrespective of whether this woman is myopic or farsighted, she is not willing to form a match with a man again and it is not possible to reach $\mu^{*}$. We have shown that $\mu^{*} \notin h^{*}\left(\mu^{\prime}\right)$.

The next result demonstrates that $g^{*}(\mu)$ and $h^{*}(\mu)$ are identical for individually rational matchings $\mu$.

Theorem 5. Let $(M, W, F, \succ)$ be a marriage problem. For every $\mu \in \mathcal{I}$ it holds that $g^{*}(\mu)=h^{*}(\mu)$.

We prove Theorem 5 using Lemma 3 that studies the case where a myopic player starts from an individually rational match and shows that along a coalitional myopicfarsighted improving path this player will never be involved in a match that is not individually rational. It also considers a farsighted player who starts from an 
individually rational match and shows that at the end matching in a coalitional myopic-farsighted improving path this player is involved in an individually rational match. Moreover, if such a player is involved in a coalitional move, then the end matching is strictly preferred to remaining single.

Using Theorem 5 and Lemma 4, which states that any element of a myopicfarsighted stable set is individually rational, we obtain an equivalence between the pairwise myopic-farsighted stable set and the coalitional myopic-farsighted stable set.

Theorem 6. Let $(M, W, F, \succ)$ be a marriage problem. A set of matchings $V \subset \mathcal{M}$ is a pairwise myopic-farsighted stable set if and only if $V \subset \mathcal{M}$ is a coalitional myopic-farsighted stable set.

Herings, Mauleon, and Vannetelbosch (2017) show the special case of Theorem 6 where all players are myopic, so $F=\emptyset$. When all players are myopic, the set of stable matchings is also shown to coincide with the core. When all players are farsighted, so $F=N$, it follows from Mauleon, Vannetelbosch and Vergote (2011) that the collection of coalitional myopic-farsighted stable sets is equal to the collection of singletons each consisting of a core element. For the case $F=N$, Theorem 6 extends the result by Mauleon, Vannetelbosch and Vergote (2011) to an equivalence with the pairwise myopic-farsighted stable set in case of individual or pairwise deviations and improvements are required to be strict for all the players involved in the deviation.

\section{Strict versus Weak Improvements}

In the definition of a pairwise myopic-farsighted improving path, a pair of players forms a match between them only if both players have a strict preference to do so. We will refer to this as the case with strict improvements. However, the alternative assumption that a pair of players forms a match between them if at least one player has a strict preference to do so can be reasonably entertained as well. We refer to this as the case with weak improvements. We already explained in detail in Section 3 that in the presence of farsighted players, the requirement of strict versus weak improvement matters, even when preferences are strict. The case with weak improvements was analyzed in Herings, Mauleon, and Vannetelbosch (2020). In this section, we compare our results to the ones in that paper. This section discusses how this alternative assumption affects our results. 
We define $h(\mu)$ as the set of all matchings that can be reached from a matching $\mu \in \mathcal{M}$ by a pairwise myopic-farsighted improving path under the condition of weak improvements. Let us refer to the stable set that results if we replace $h^{*}$ by $h$ in Definition 2 as the weak pairwise myopic-farsighted stable set.

Let $g(\mu)$ denote the matchings $\mu^{\prime} \in \mathcal{M}$ that can be reached from $\mu \in \mathcal{M}$ by a coalitional myopic-farsighted improving path under the condition of weak improvements, meaning that all players in the coalition improve weakly and at least one player strictly. Replacing $g^{*}$ by $g$ in Definition 6, leads to the weak coalitional myopic-farsighted stable set.

In Example 1, we have shown that $\bar{\mu} \in h^{*}\left(\mu^{\mathrm{W}}\right)$ while $\mu^{\mathrm{W}} \notin h^{*}(\bar{\mu})$. Replacing strict by weak improvements, we still have that $\bar{\mu} \in h\left(\mu^{\mathrm{W}}\right)$, but now we also have that $\mu^{\mathrm{W}} \in h(\bar{\mu})$ despite the fact that all women are farsighted and that $\bar{\mu}$ is strictly preferred by two out of three women to $\mu^{\mathrm{W}}$, whereas the third woman is indifferent. Moreover, Example 2 still holds and $V=\{\bar{\mu}\}$ is a weak pairwise myopic-farsighted stable set. However, in Example 3, on top of $\{\bar{\mu}\}$ now also $\left\{\mu^{\mathrm{W}}\right\}$ emerges as a weak pairwise myopic-farsighted stable set.

The analogue of Theorem 1 for weak improvements has not been studied so far, but can be shown along the same lines. In case of weak improvements, the analogue of Theorem 2, $\left\{\mu^{\mathrm{W}}\right\}$ is a weak pairwise myopic-farsighted stable set holds even when $\mu^{\mathrm{W}}$ is $W$-dominated by a matching in $\mathcal{I}$. It is therefore easier to sustain $\mu^{\mathrm{W}}$ as the outcome when only weak improvements are required. The analogue of Theorem 3 does not hold. There are marriage problems satisfying the conditions of Theorem 3 such that core elements different from $\left\{\mu^{\mathrm{W}}\right\}$ form a singleton weak pairwise myopicfarsighted stable set. The next example presents a matching problem where both $\left\{\mu^{\mathrm{W}}\right\}$ and $\left\{\mu^{\mathrm{M}}\right\}$ are weak pairwise myopic-farsighted stable sets, which implies that it is possible to reach the man-optimal stable matching $\mu^{\mathrm{M}}$ from $\mu^{\mathrm{W}}$.

Example 5. Consider the marriage problem $(M, W, F, \succ)$, where $M=\left\{m_{1}, m_{2}, m_{3}\right\}$ and $W=\left\{w_{1}, w_{2}, w_{3}\right\}$ and the preferences of the players are as follows:

\begin{tabular}{|c|c|c|c|c|}
\hline$m_{1}$ & $\underline{m_{2}}$ & $\underline{m_{3}}$ & $\underline{w_{1}}$ & $\underline{w_{2}}$ \\
\hline$w_{1}$ & $w_{3}$ & $w_{1}$ & $m_{1}$ & $m_{2}$ \\
\hline$w_{2}$ & $w_{1}$ & $w_{2}$ & $m_{2}$ & $m_{3}$ \\
\hline$w_{3}$ & $w_{2}$ & $w_{3}$ & $m_{3}$ & $m_{1}$ \\
\hline
\end{tabular}

We assume $F=W$, so all women are farsighted and all men are myopic. Using the deferred acceptance algorithm of Gale and Shapley (1962) with men proposing and 
with women proposing, it is immediate that the man-optimal and woman-optimal stable matchings are given by

$$
\begin{array}{ll}
\mu^{\mathrm{M}}\left(m_{1}\right)=w_{1}, & \mu^{\mathrm{W}}\left(m_{1}\right)=w_{1}, \\
\mu^{\mathrm{M}}\left(m_{2}\right)=w_{3}, & \mu^{\mathrm{W}}\left(m_{2}\right)=w_{2}, \\
\mu^{\mathrm{M}}\left(m_{3}\right)=w_{2} . & \mu^{\mathrm{W}}\left(m_{3}\right)=w_{3},
\end{array}
$$

We first argue that $\mu^{\mathrm{M}} \in h\left(\mu^{\mathrm{W}}\right)$. To verify this assertion, consider the weak pairwise myopic-farsighted improving path $\mu_{0}, \ldots, \mu_{4}$ with $\mu_{0}=\mu^{\mathrm{W}}$ and $\mu_{4}=\mu^{\mathrm{M}}$, where $\mu_{1}=\mu_{0}+\left(m_{2}, w_{1}\right), \mu_{2}=\mu_{1}+\left(m_{3}, w_{2}\right), \mu_{3}=\mu_{2}+\left(m_{2}, w_{3}\right)$, and $\mu_{4}=$ $\mu_{3}+\left(m_{1}, w_{1}\right)$. For an illustration, see Figure 2 .

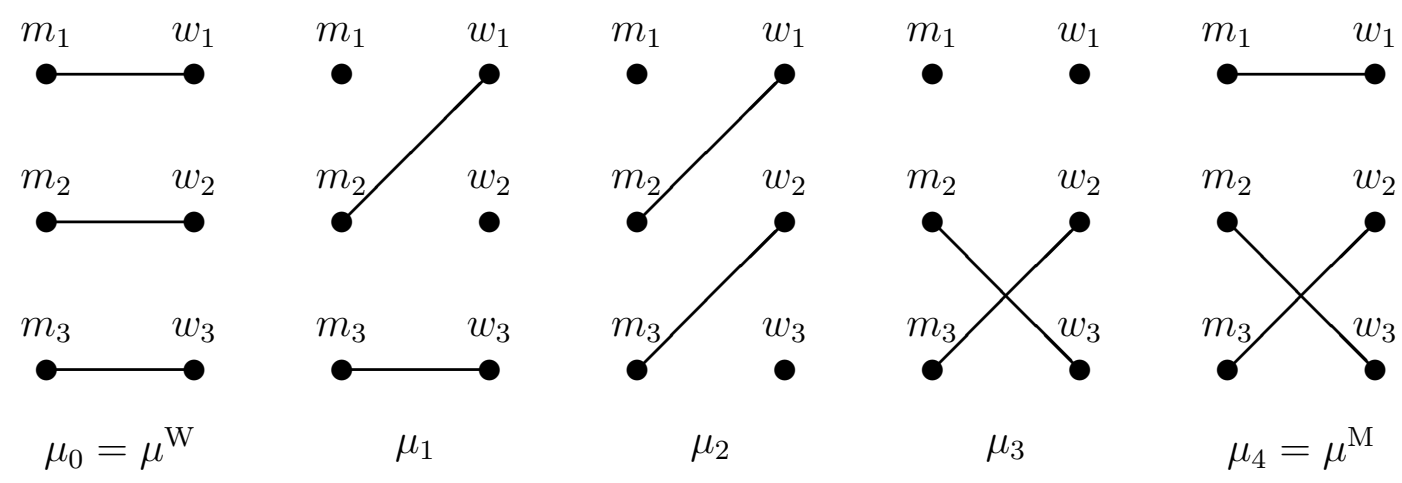

Figure 2: Myopic-farsighted improving path in Example 5 to move from $\mu^{\mathrm{W}}$ to $\mu^{\mathrm{M}}$.

Indeed, $m_{2}$ strictly prefers $\mu_{1}\left(m_{2}\right)=w_{1}$ to $\mu_{0}\left(m_{2}\right)=w_{2}$ and the farsighted woman $w_{1}$ is indifferent between $\mu_{4}$ and $\mu_{0}$. Since now at $\mu_{1}$ woman $w_{2}$ has become unmatched, she is willing to form a link with $m_{3}$, her partner in the end matching of the sequence, moving from $\mu_{1}$ to $\mu_{2}$. Since $\mu_{2}\left(m_{3}\right)=w_{2} \succ_{m_{3}} w_{3}=\mu_{1}\left(m_{3}\right)$, this is also a myopic improvement for $m_{3}$. At $\mu_{2}$ woman $w_{3}$ is unmatched, so she is willing to team up with $m_{2}$, her partner in the end matching of the sequence, leading to the matching $\mu_{3}$. Since $\mu_{3}\left(m_{2}\right)=w_{3} \succ_{m_{2}} w_{1}=\mu_{2}\left(m_{2}\right)$, this is also a myopic improvement for $m_{2}$. Man $m_{1}$ and woman $w_{1}$ are both single at $\mu_{3}$ and are both happy to marry, which moves them to the end matching $\mu_{4}=\mu^{\mathrm{M}}$.

Consider next any matching $\mu \in \mathcal{M} \backslash\left\{\mu^{\mathrm{M}}, \mu^{\mathrm{W}}\right\}$. We argue that $\mu^{\mathrm{M}} \in h(\mu)$ by constructing a weak pairwise myopic-farsighted improving path $\mu_{0}, \ldots, \mu_{L}$ with $\mu_{0}=\mu$ and $\mu_{L}=\mu^{\mathrm{M}}$.

Assume first that $\mu\left(m_{1}\right) \neq w_{1}$. We define $\mu_{1}=\mu_{0}+\left(m_{1}, w_{1}\right)$. Since $w_{1}$ is the best partner for $m_{1}$, this is clearly a myopic improvement for $m_{1}$. Since $m_{1}$ is the 
best partner for $w_{1}$ and $\mu_{L}\left(w_{1}\right)=\mu^{\mathrm{M}}\left(w_{1}\right)=m_{1}$, this is a farsighted improvement for $w_{1}$.

If $\mu_{1}=\mu^{\mathrm{M}}$, then we have shown that $\mu^{\mathrm{M}} \in h(\mu)$.

If $\mu_{1}=\mu^{\mathrm{W}}$, then following the weak pairwise myopic-farsighted improving path from $\mu^{\mathrm{W}}$ to $\mu^{\mathrm{M}}$ constructed at the beginning of the example, we also have $\mu^{\mathrm{M}} \in h(\mu)$.

If $\mu_{1} \in \mathcal{M} \backslash\left\{\mu^{\mathrm{M}}, \mu^{\mathrm{W}}\right\}$, then $w_{2}$ or $w_{3}$ is single under $\mu_{1}$. If $w_{2}$ is single, then let her marry $m_{3}$ and move to $\mu_{2}=\mu_{1}+\left(m_{3}, w_{2}\right)$. Since at $\mu_{1}$ man $m_{3}$ is not married to $w_{1}$, this is a myopic improvement for $m_{3}$. It is also clearly a farsighted improvement for $w_{2}$. If $w_{2}$ is not single, but $w_{3}$ is, then let her marry $m_{2}$ and move to $\mu_{2}=\mu_{1}+\left(m_{2}, w_{3}\right)$. Since $w_{3}$ is the preferred partner of $m_{2}$, this is clearly a myopic improvement for $m_{2}$. It is also clearly a farsighted improvement for $w_{3}$. Either $\mu_{2}=\mu^{\mathrm{M}}$ and we are done, or $\mu^{2}$ consists of two matched pairs $\left(m_{1}, w_{1}\right)$ and $\left(m_{3}, w_{2}\right)$, both being part of $\mu^{\mathrm{M}}$, and two single players, $m_{2}$ and $w_{3}$. In this case, we form the missing pair $\left(m_{2}, w_{3}\right)$ from $\mu^{\mathrm{M}}$ and move from $\mu_{2}$ to $\mu_{3}=\mu^{\mathrm{M}}$. This completes the construction of the weak pairwise myopic-farsighted improving path to $\mu^{\mathrm{M}}$ for the case $\mu\left(m_{1}\right) \neq w_{1}$.

Assume next that $\mu\left(m_{1}\right)=w_{1}$. We can then proceed with the weak pairwise myopic-farsighted improving path starting from $\mu_{1}$ as constructed in the previous paragraph, with $\mu_{1}$ being replaced by $\mu$.

The singleton set $V=\left\{\mu^{\mathrm{M}}\right\}$ trivially satisfies internal stability. Since we have shown that $\mu^{\mathrm{M}} \in h(\mu)$ for every $\mu \neq \mu^{\mathrm{M}}$, it also satisfies external stability. It follows that $V=\left\{\mu^{\mathrm{M}}\right\}$ is a weak pairwise myopic-farsighted stable set.

The analogue of Theorem 4 does not hold. The set $\left\{\mu^{\mathrm{W}}\right\}$ is a weak pairwise myopic-farsighted stable set even when it is $W$-dominated by an individually rational matching $\bar{\mu} \in \mathcal{I}$.

Regarding the results in Section 5, we now present an example to demonstrate that it is not possible to obtain the analogue of Theorem 5 for the case of weak improvements.

Example 6. Consider the marriage problem $(M, W, F, \succ)$ with $M=\left\{m_{1}, m_{2}, m_{3}\right\}$ and $W=\left\{w_{1}, w_{2}, w_{3}\right\}$. Assume $F=\left\{w_{1}\right\}$. The preferences of the players are as 
follows, where only the individually rational part is specified.

$\begin{array}{llllll}\frac{m_{1}}{w_{1}} & \frac{m_{2}}{w_{2}} & \frac{m_{3}}{w_{3}} & \frac{w_{1}}{m_{1}} & \frac{w_{2}}{m_{1}} & \frac{w_{3}}{m_{2}} \\ w_{2} & w_{3} & & & m_{2} & m_{3}\end{array}$

In the figure it is shown that $\mu_{4} \in g\left(\mu_{0}\right)$.

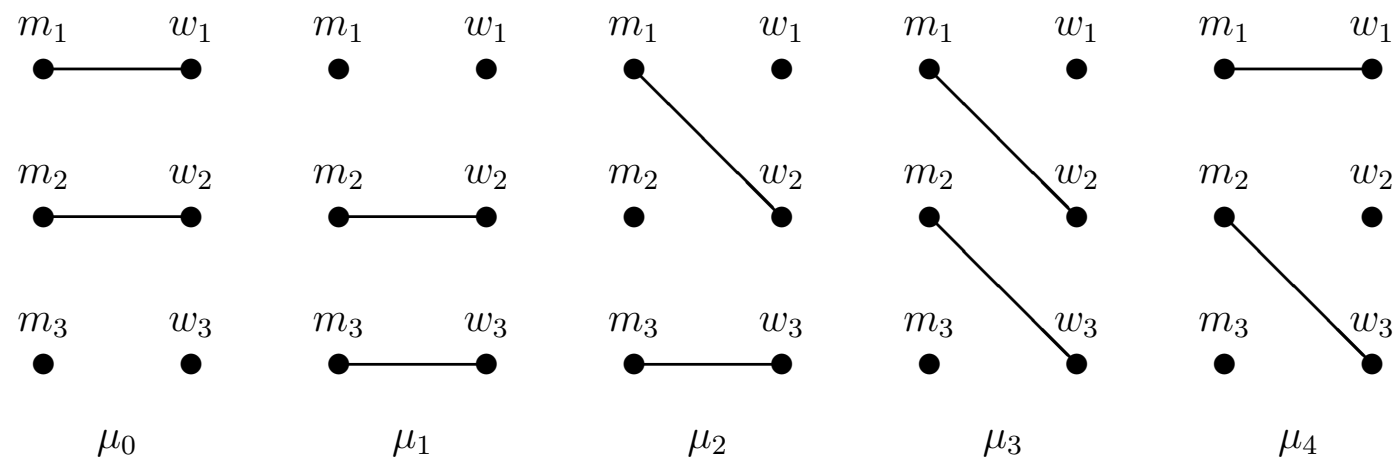

Figure 3: Myopic-farsighted improving path to move from $\mu_{0}$ to $\mu_{4}$.

The move from $\mu_{0}$ to $\mu_{1}$ is performed by the coalition $\left\{m_{3}, w_{1}, w_{3}\right\}$. A link is formed by the myopic players $m_{3}$ and $w_{3}$ and both improve. The link $\left(m_{1}, w_{1}\right)$ is deleted by the farsighted $w_{1}$, who doesn't care. The move from $\mu_{1}$ to $\mu_{2}$ is made by coalition $\left\{m_{1}, w_{2}\right\}$. Both players are myopic and improve. The move from $\mu_{2}$ to $\mu_{3}$ is performed by the coalition $\left\{m_{2}, w_{3}\right\}$. Both players are myopic and improve. The move from $\mu_{3}$ to $\mu_{4}$ is carried out by coalition $\left\{m_{1}, w_{1}\right\}$. Both players are improving. This shows that $\mu_{4} \in g\left(\mu_{0}\right)$.

We now argue that $\mu_{4} \notin h\left(\mu_{0}\right)$. Suppose $\mu_{0}^{\prime}, \ldots, \mu_{L}^{\prime}$ is a pairwise myopic-farsighted improving path with $\mu_{0}^{\prime}=\mu_{0}$ and $\mu_{L}^{\prime}=\mu_{4}$. At $\mu_{0}^{\prime}$, woman $w_{1}$ is farsighted and indifferent to the match at $\mu_{L}^{\prime}$. She is only willing to move if one of the myopic men would strictly improve marrying her. No man wants to do so, so $w_{1}$ cannot move at $\mu_{0}^{\prime}$. At $\mu_{0}^{\prime}$, the myopic men $m_{1}$ and $m_{2}$ are at their best match, so not willing to move. Although the myopic woman $w_{2}$ is willing to marry $m_{1}, m_{1}$ is not willing to marry $w_{2}$, so $w_{2}$ cannot move. The only possibility is that the match $\left(m_{3}, w_{3}\right)$ is formed, so $\mu_{1}^{\prime}$ is the matching where the pairs $\left(m_{1}, w_{1}\right),\left(m_{2}, w_{2}\right)$, and $\left(m_{3}, w_{3}\right)$ are all formed. It is easily verified that no moves away from $\mu_{1}^{\prime}$ are possible. Since 
$\mu_{1}^{\prime} \neq \mu_{4}$, this shows that $\mu_{4} \notin h\left(\mu_{0}\right)$.

\section{Conclusion}

We use the pairwise myopic-farsighted stable set to study marriage problems where myopic and farsighted players interact. We assume that players can only deviate if they improve strictly. For homogeneous cases, where either all players are myopic or all players are farsighted, every element of a myopic-farsighted stable set is a core element and every core element belongs to some myopic-farsighted stable set. We cannot discriminate between different core elements and matchings outside of the core cannot result. The same result holds for heterogeneous cases when preferences satisfy the top-coalition property.

But in general, the interaction between myopic and farsighted players leads to selection of core elements and to matchings that do not belong to the core. When all women are farsighted and all men are myopic, then the only subset of the core that can be a pairwise myopic-farsighted stable set is the singleton consisting of the woman-optimal stable matching. In case the woman-optimal stable matching is dominated from the woman point of view by an individually rational matching, then the woman-optimal stable matching cannot be a pairwise myopic-farsighted stable set and the core and the pairwise myopic-farsighted stable set provide different predictions. These results are robust to the case where coalitional deviations are allowed, since the pairwise myopic-farsighted stable set is shown to be equivalent to the coalitional pairwise myopic-farsighted stable set.

Herings, Mauleon and Vannetelbosch (2020) study the weak pairwise myopicfarsighted stable set, which results when players can deviate if none of them is worse off and at least one is strictly better off. Even though we assume strict preferences, the presence of farsighted players makes the two notions different and, indeed, we obtain quite different results. In the weak improvements case, even if all women are farsighted and all men are myopic, the woman-optimal stable matching is always a weak pairwise myopic-farsighted stable set and, depending on the preferences, it is possible to sustain the man-optimal stable matching. Moreover, coalitional deviations are not identical to pairwise deviations.

In this paper, the evidence against the core when myopic and farsighted play- 
ers interact is stronger. There is more scope for the farsighted side of a market to obtain a matching that dominates their optimal matching in the core and thereby more scope for exploiting the myopic side of the market.

\section{Acknowledgments}

We thank Rajiv Vohra for stimulating discussions on this subject. Ana Mauleon and Vincent Vannetelbosch are, respectively, Research Director and Senior Research Associate of the National Fund for Scientific Research (FNRS). Financial support from the MSCA ITN Expectations and Social Influence Dynamics in Economics (ExSIDE), Grant No721846 (1/9/2017-31/8/2020), from the Belgian French speaking community ARC project $n^{\circ} 15 / 20-072$ of Saint-Louis University - Brussels and from the Fonds de la Recherche Scientifique - FNRS research grant T.0143.18 is gratefully acknowledged.

\section{Appendix}

\section{Proof of Theorem 1.}

Let $\left\{S_{1}^{*}, S_{2}^{*}, \ldots, S_{k}^{*}\right\}$ be the top-coalition partition and $\mu^{*}$ the induced matching, so $C=\left\{\mu^{*}\right\}$.

First, we show that $V=\left\{\mu^{*}\right\}$ is a pairwise myopic-farsighted stable set by showing that for every $\mu \in \mathcal{M} \backslash\left\{\mu^{*}\right\}$ we have that $\mu^{*} \in h^{*}(\mu)$.

Let $\mu \in \mathcal{M} \backslash\left\{\mu^{*}\right\}$ be given. We construct a finite sequence of distinct matchings $\mu_{0}, \ldots, \mu_{L}$ with $\mu_{0}=\mu$ and $\mu_{L}=\mu^{*}$ such that, for every $\ell \in\{0, \ldots, L-1\}$, there is a coalition $S_{\ell} \in \mathcal{N}$ with cardinality at most two that can enforce $\mu_{\ell+1}$ from $\mu_{\ell}$ and

$$
\begin{array}{ll}
\mu_{\ell+1}(i) \succ_{i} \mu_{\ell}(i), & \text { if } i \in S_{\ell} \backslash F, \\
\mu_{L}(i) \succ_{i} \mu_{\ell}(i), & \text { if } i \in S_{\ell} \cap F .
\end{array}
$$

If the players in $S_{1}^{*}$ are not matched under $\mu$, then let $S_{1}^{*}$ move from $\mu_{0}$ to $\mu_{1}$, with $\mu_{1}\left(S_{1}^{*}\right)=S_{1}^{*}$. Obviously, it holds that $\mu_{1}(i) \succ_{i} \mu_{0}(i)$ if $i \in S_{1}^{*} \backslash F$, and $\mu_{L}(i) \succ_{i} \mu_{0}(i)$ if $i \in S_{1}^{*} \cap F$. Next, if the players in $S_{2}^{*}$ are not matched under $\mu_{1}$, then let $S_{2}^{*}$ move from $\mu_{1}$ to $\mu_{2}$, with $\mu_{2}\left(S_{2}^{*}\right)=S_{2}^{*}$. Since $S_{2}^{*}$ is a top coalition of $N \backslash S_{1}^{*}$ and $\mu_{1}\left(S_{1}^{*}\right)=S_{1}^{*}$, it holds that $\mu_{2}(i) \succ_{i} \mu_{1}(i)$ if $i \in S_{2}^{*} \backslash F$, and $\mu_{L}(i) \succ_{i} \mu_{1}(i)$ if $i \in S_{2}^{*} \cap F$. By repeating this argument, we obtain the matching $\mu^{*}$ after a maximum of $k$ steps. We have shown that $\mu^{*} \in h^{*}(\mu)$. 
Second, we show that $V=\left\{\mu^{*}\right\}$ is the unique pairwise myopic-farsighted stable set. We start by showing that $h^{*}\left(\mu^{*}\right)=\emptyset$.

Suppose there exists a matching $\mu \in \mathcal{M}$ such that $\mu \in h^{*}\left(\mu^{*}\right)$. Then there is a finite sequence of distinct matchings $\mu_{0}, \ldots, \mu_{L}$ with $\mu_{0}=\mu^{*}$ and $\mu_{L}=\mu$ such that, for every $\ell \in\{0, \ldots, L-1\}$, there is a coalition $S_{\ell} \in \mathcal{N}$ with cardinality at most two that can enforce $\mu_{\ell+1}$ from $\mu_{\ell}$ and

$$
\begin{array}{ll}
\mu_{\ell+1}(i) \succ_{i} \mu_{\ell}(i), & \text { if } i \in S_{\ell} \backslash F, \\
\mu_{L}(i) \succ_{i} \mu_{\ell}(i), & \text { if } i \in S_{\ell} \cap F .
\end{array}
$$

We use induction to show that, for every $j \in\{1, \ldots, k\}, S_{j}^{*} \cap\left(S_{0} \cup \cdots \cup S_{L-1}\right)=\emptyset$, which leads to the desired contradiction. Since $S_{1}^{*}$ is a top-coalition of $N$ and $\mu^{*}\left(S_{1}^{*}\right)=S_{1}^{*}$, it clearly holds that $S_{1}^{*} \cap\left(S_{0} \cup \cdots \cup S_{L-1}\right)=\emptyset$.

Assume that, for some $j \in\{1, \ldots, k-1\},\left(S_{1}^{*} \cup \cdots \cup S_{j}^{*}\right) \cap\left(S_{0} \cup \cdots \cup S_{L-1}\right)=\emptyset$. It follows that, for every $\ell \in\{0, \ldots, L\}$, for every $i \in\left(S_{1}^{*} \cup \cdots \cup S_{j}^{*}\right), \mu_{\ell}(i)=\mu^{*}(i)$. Since $S_{j+1}^{*}$ is a top coalition of $N \backslash\left(S_{1}^{*} \cup \cdots \cup S_{j}^{*}\right)$, it follows that, for every $i \in S_{j+1}^{*}$, $\mu_{1}(i) \preceq_{i} \mu_{0}(i)$ and $\mu_{L}(i) \preceq_{i} \mu_{0}(i)$, so $i \notin S_{0}$ and $\mu_{1}(i)=\mu^{*}(i)$. Repeating this argument, it follows that $S_{j+1}^{*} \cap\left(S_{0} \cup \cdots \cup S_{L-1}\right)=\emptyset$.

We have shown that $h^{*}\left(\mu^{*}\right)=\emptyset$.

By external stability as in Definition 2 it follows that $\mu^{*} \in V$. Since $\mu^{*} \in h^{*}(\mu)$ for all $\mu \in \mathcal{M} \backslash\left\{\mu^{*}\right\}$, internal stability as in Definition 2 implies that $V=\left\{\mu^{*}\right\}$ is the unique pairwise myopic-farsighted stable set.

Lemma 1. Let $(M, W, F, \succ)$ be a marriage problem with $F \supset W$. For every $\mu \in$ $\mathcal{M} \backslash\left\{\mu^{\mathrm{W}}\right\}$ such that $W(\mu)=\emptyset$ it holds that $\mu^{\mathrm{W}} \in h^{*}(\mu)$.

Proof. Let $\mu \in \mathcal{M} \backslash\left\{\mu^{\mathrm{W}}\right\}$ be a matching such that $W(\mu)=\emptyset$. We construct a pairwise myopic-farsighted improving path $\mu_{0}, \ldots, \mu_{L}$ from $\mu_{0}=\mu$ to $\mu_{L}=\mu^{\mathrm{W}}$. Let

$$
W^{1}=\left\{w \in W \mid \mu^{\mathrm{W}}(w) \succ_{w} \mu(w) \text { and } \mu(w) \in M\right\}
$$

be the, possibly empty, set of women who strictly prefer $\mu^{\mathrm{W}}(w)$ to $\mu(w)$ and who are married at $\mu$. Let $k^{1}$ be the cardinality of $W^{1}$. Take an arbitrary order of the women in $W^{1}$, say $w_{0}, \ldots, w_{k^{1}-1}$.

For $\ell \in\left\{0, \ldots, k^{1}-1\right\}$, we define the matching $\mu_{\ell+1}=\mu_{\ell}-\left(\mu_{\ell}\left(w_{\ell}\right), w_{\ell}\right)$, so the $k^{1}$ women in $W^{1}$ sequentially destroy their matches. We show that the sequence 
of matchings $\mu_{0}, \ldots, \mu_{k^{1}}$ is the first part of a pairwise myopic-farsighted improving path from $\mu$ to $\mu^{\mathrm{W}}$ by showing that for every $\ell \in\left\{0, \ldots, k^{1}-1\right\}$ we have $\mu_{L}\left(w_{\ell}\right)=$ $\mu^{\mathrm{W}}\left(w_{\ell}\right) \succ_{w_{\ell}} \mu_{\ell}\left(w_{\ell}\right)$. This follows since $\mu_{\ell}\left(w_{\ell}\right)=\mu\left(w_{\ell}\right)$ and by the definition of $W^{1}$.

At $\mu_{k^{1}}$ every woman is either single or married to her partner at $\mu^{\mathrm{W}}$. It then follows that at $\mu_{k^{1}}$ every man is either single or married to his partner at $\mu^{\mathrm{W}}$. Let

$$
W^{2}=\left\{w \in W \mid \mu^{\mathrm{W}}(w) \succ_{w} \mu_{k^{1}}(w)\right\}
$$

be the set of women that strictly prefer the match at $\mu^{\mathrm{W}}$ to being single. Let $k^{2}$ be the cardinality of the set $W^{2}$. Take an arbitrary order of the women in $W^{2}$, say $w_{0}, \ldots, w_{k^{2}-1}$. For $\ell=0, \ldots, k^{2}-1$, we define $m_{\ell}=\mu^{\mathrm{W}}\left(w_{\ell}\right)$.

For $\ell=0, \ldots, k^{2}-1$, we define the matching $\mu_{k^{1}+\ell+1}=\mu_{k^{1}+\ell}+\left(m_{\ell}, w_{\ell}\right)$, so the $k^{2}$ women in $W^{2}$ sequentially marry their partners at $\mu^{\mathrm{W}}$. It holds that $\mu_{L}=$ $\mu_{k^{1}+k^{2}}=\mu^{\mathrm{W}}$. Observe that the sequence of matchings $\mu_{k^{1}}, \ldots, \mu_{k^{1}+k^{2}}$ is the final part of a pairwise myopic-farsighted improving path from $\mu$ to $\mu^{\mathrm{W}}$ since it holds that, for every $\ell \in\left\{0, \ldots, k^{2}-1\right\}$,

$$
\begin{aligned}
\mu_{k^{1}+\ell+1}\left(m_{\ell}\right) & =\mu^{\mathrm{W}}\left(m_{\ell}\right) \succ_{m_{\ell}} m_{\ell}=\mu_{k^{1}+\ell}\left(m_{\ell}\right), \quad \text { if } m_{\ell} \in M \backslash F, \\
\mu_{L}\left(m_{\ell}\right) & =\mu^{\mathrm{W}}\left(m_{\ell}\right) \succ_{m_{\ell}} m_{\ell}=\mu_{k^{1}+\ell}\left(m_{\ell}\right), \quad \text { if } m_{\ell} \in F, \\
\mu_{L}\left(w_{\ell}\right) & =\mu^{\mathrm{W}}\left(w_{\ell}\right) \succ_{w_{\ell}} w_{\ell}=\mu_{k^{1}+\ell}\left(w_{\ell}\right) .
\end{aligned}
$$

Lemma 2. Let $(M, W, F, \succ)$ be a marriage problem with $F \supset W$. Assume that $\mu^{\mathrm{W}}$ is not $W$-dominated by a matching in $\mathcal{I}$. For every $\mu \in \mathcal{M}$ such that $W(\mu) \neq \emptyset$ it holds that $\mu^{\mathrm{W}} \in h^{*}(\mu)$.

Proof. Let $\mu \in \mathcal{M}$ be a matching such that $W(\mu) \neq \emptyset$. We construct a pairwise myopic-farsighted improving path $\mu_{0}, \ldots, \mu_{L}$ from $\mu_{0}=\mu$ to $\mu_{L}=\mu^{\mathrm{W}}$. Let

$$
W^{1}=\left\{w \in \mu(M) \mid \mu(w) \succ_{\mu(w)} w \text { or } w \succ_{w} \mu(w)\right\}
$$

be the, possibly empty, set of women that are involved in a match that is not individually rational for at least one of the partners and denote the cardinality of $W^{1}$ by $k^{1}$. Take an arbitrary order of the women in $W^{1}$, say $w_{0}, \ldots, w_{k^{1}-1}$. For $\ell \in\left\{0, \ldots, k^{1}-1\right\}$, we define $m_{\ell}=\mu\left(w_{\ell}\right)$ to be the man married to $w_{\ell}$ at $\mu$ and we define the matching $\mu_{\ell+1}=\mu_{\ell}-\left(m_{\ell}, w_{\ell}\right)$, so the player who is involved in a match under $\mu$ that is not individually rational destroys his or her match. We argue that 
the sequence of matchings $\mu_{0}, \ldots, \mu_{k^{1}}$ is the first part of a pairwise myopic-farsighted improving path from $\mu$ to $\mu^{\mathrm{W}}$. Notice that $\mu_{k^{1}} \in \mathcal{I}$.

Let some $\ell \in\left\{0, \ldots, k^{1}-1\right\}$ be given. It holds that $m_{\ell} \succ_{m_{\ell}} w_{\ell}$ or $w_{\ell} \succ_{w_{\ell}} m_{\ell}$. In the former case, we have that

$$
\begin{aligned}
\mu_{\ell+1}\left(m_{\ell}\right) & =m_{\ell} \succ_{m_{\ell}} \mu_{\ell}\left(m_{\ell}\right), & & \text { if } m_{\ell} \in M \backslash F, \\
\mu_{L}\left(m_{\ell}\right) & =\mu^{\mathrm{W}}\left(m_{\ell}\right) \succeq_{m_{\ell}} m_{\ell} \succ_{m_{\ell}} \mu_{\ell}\left(m_{\ell}\right), & & \text { if } m_{\ell} \in F,
\end{aligned}
$$

whereas in the latter case it holds that

$$
\mu_{L}\left(w_{\ell}\right)=\mu^{\mathrm{W}}\left(w_{\ell}\right) \succeq_{w_{\ell}} w_{\ell} \succ_{w_{\ell}} \mu_{\ell}\left(w_{\ell}\right)
$$

so the conditions of Definition 1 are satisfied.

Let

$$
W^{2}=\left\{w \in W\left(\mu_{k^{1}}\right) \mid \mu_{k^{1}}(w) \in F\right\}
$$

be the, possibly empty, set of women that prefer their match at $\mu_{k^{1}}$ to the one at $\mu^{\mathrm{W}}$ and that are matched to a farsighted man. We denote the cardinality of $W^{2}$ by $k^{2}$ and take an arbitrary order of the women in $W^{2}$, say $w_{0}, \ldots, w_{k^{2}-1}$. For $\ell \in\left\{0, \ldots, k^{2}-1\right\}$, we define $m_{\ell}=\mu_{k^{1}}\left(w_{\ell}\right)$ to be the man married to $w_{\ell}$ at $\mu_{k^{1}}$ and we define the matching $\mu_{k^{1}+\ell+1}=\mu_{k^{1}+\ell}-\left(m_{\ell}, w_{\ell}\right)$, so man $m_{\ell}$ destroys his match with $w_{\ell}$. We argue that the sequence of matchings $\mu_{k^{1}}, \ldots, \mu_{k^{1}+k^{2}}$ is the second part of a pairwise myopic-farsighted improving path from $\mu$ to $\mu^{\mathrm{W}}$. Notice that $\mu_{k^{1}+k^{2}} \in \mathcal{I}$.

Let some $\ell \in\left\{0, \ldots, k^{2}-1\right\}$ be given. Since $m_{\ell} \succ_{w_{\ell}} \mu^{\mathrm{W}}\left(w_{\ell}\right)$ and $\mu^{\mathrm{W}}$ is a core element, it holds that

$$
\mu_{L}\left(m_{\ell}\right)=\mu^{\mathrm{W}}\left(m_{\ell}\right) \succ_{m_{\ell}} w_{\ell}=\mu_{k^{1}+\ell}\left(m_{\ell}\right),
$$

so the conditions of Definition 1 are satisfied.

Let

$$
W^{3}=W\left(\mu_{k^{1}+k^{2}}\right)
$$

be the set of women that strictly prefer $\mu_{k^{1}+k^{2}}(w)$ to $\mu^{\mathrm{W}}(w)$. By construction of $\mu_{k^{1}+k^{2}}$ it holds that all men in the set $\mu_{k^{1}+k^{2}}\left(W^{3}\right)$, i.e., the men who are married to a woman in $W^{3}$, are myopic. If $W^{3}=\emptyset$, then the matching $\mu_{k^{1}+k^{2}}$ is equal to $\mu^{\mathrm{W}}$ or satisfies the assumptions of Lemma 1. In the former case we are done, in the latter case we proceed as in the proof of Lemma 1 to complete the construction of 
the pairwise myopic-farsighted improving path leading to $\mu^{\mathrm{W}}$. It remains to consider the situation where $W^{3} \neq \emptyset$.

We consider two cases.

Case 1. $\mu^{\mathrm{W}}\left(W^{3}\right)=\mu_{k^{1}+k^{2}}\left(W^{3}\right)$.

In this case, we sequentially marry all women outside $W^{3}$ to their partner at $\mu^{\mathrm{W}}$, resulting in a matching $\bar{\mu}$. The matching $\bar{\mu}$ belongs to $\mathcal{I}$ and $W$-dominates the matching $\mu^{\mathrm{W}}$, leading to a contradiction with the assumptions of the lemma. This case can therefore not occur.

Case 2. $\mu^{\mathrm{W}}\left(W^{3}\right) \neq \mu_{k^{1}+k^{2}}\left(W^{3}\right)$.

For $\ell \in\{0,1, \ldots\}$, whenever $\mu^{\mathrm{W}}\left(W^{3+\ell}\right) \neq \mu_{k^{1}+k^{2}+\ell}\left(W^{3+\ell}\right)$, select some $m_{\ell} \in$ $\mu_{k^{1}+k^{2}+\ell}\left(W^{3+\ell}\right) \backslash \mu^{\mathrm{W}}\left(W^{3+\ell}\right)$ and let $w_{\ell}=\mu^{\mathrm{W}}\left(m_{\ell}\right)$. This selection of $m_{\ell}$ is possible since all women in $W^{3+\ell}$ are married at $\mu_{k^{1}+k^{2}+\ell}$, so the cardinality of $\mu_{k^{1}+k^{2}+\ell}\left(W^{3+\ell}\right)$ is greater than or equal to the cardinality of $\mu^{\mathrm{W}}\left(W^{3+\ell}\right)$.

We argue that $\left(m_{\ell}, w_{\ell}\right)$ blocks $\mu_{k^{1}+k^{2}+\ell}$. Let $w^{\prime}=\mu_{k^{1}+k^{2}+\ell}\left(m_{\ell}\right)$. By definition of $W^{3+\ell}, w^{\prime}$ prefers $m_{\ell}$ to $\mu^{\mathrm{W}}\left(w^{\prime}\right)$ and $m_{\ell}$ prefers $\mu^{\mathrm{W}}\left(m_{\ell}\right)=w_{\ell}$ to $w^{\prime}$, because otherwise $\left(m_{\ell}, w_{\ell}\right)$ blocks $\mu^{\mathrm{W}}$. Since $m_{\ell} \notin \mu^{\mathrm{W}}\left(W^{3+\ell}\right)$, we have that $w_{\ell} \notin W^{3+\ell}$, so $w_{\ell}$ prefers $m_{\ell}=\mu^{\mathrm{W}}\left(w_{\ell}\right)$ to $\mu_{k^{1}+k^{2}+\ell}\left(w_{\ell}\right)$. We have shown that $\left(m_{\ell}, w_{\ell}\right)$ blocks $\mu_{k^{1}+k^{2}+\ell}$.

We define the matching $\mu_{k^{1}+k^{2}+\ell+1}=\mu_{k^{1}+k^{2}+\ell}+\left(m_{\ell}, w_{\ell}\right)$ and $W^{3+\ell+1}=W\left(\mu_{k^{1}+k^{2}+\ell+1}\right)$. Let $k^{3}$ denote the cardinality of $W\left(\mu_{k^{1}+k^{2}}\right)$. For every $\ell \geq 0$, at $\mu_{k^{1}+k^{2}+\ell} \operatorname{man} m_{\ell}$ is married to a woman in $W^{3+\ell}$ and is married to a woman not in $W^{3+\ell}$ at $\mu_{k^{1}+k^{2}+\ell+1}$, it follows that the cardinality of the set $W^{3+\ell}$ is strictly decreasing in $\ell$. By the argument of Case 1, it cannot occur that $\mu^{\mathrm{W}}\left(W^{3+\ell}\right)=\mu_{k^{1}+k^{2}+\ell}\left(W^{3+\ell}\right)$. Therefore, we have that $W^{3+k^{3}}=\emptyset$.

We argue that the sequence of matchings $\mu_{k^{1}+k^{2}}, \ldots, \mu_{k^{1}+k^{2}+k^{3}}$ is the third part of a pairwise myopic-farsighted improving path from $\mu$ to $\mu^{\mathrm{W}}$.

Let some $\ell \in\left\{0, \ldots, k^{3}-1\right\}$ be given. Since the set of men $\mu_{k^{1}+k^{2}+\ell}\left(W^{3+\ell}\right)$ is a subset of $\mu_{k^{1}+k^{2}}\left(W^{3}\right)$, it holds that $m_{\ell}$ is myopic. Since $\left(m_{\ell}, w_{\ell}\right)$ blocks $\mu_{k^{1}+k^{2}+\ell}$, it follows that

$$
\mu_{k^{1}+k^{2}+\ell+1}\left(m_{\ell}\right) \succ_{m_{\ell}} \mu_{k^{1}+k^{2}+\ell}\left(m_{\ell}\right)
$$

It holds that $w_{\ell} \in W \backslash W^{3+\ell}$ and $\mu_{k^{1}+k^{2}+\ell}\left(m_{\ell}\right) \neq w_{\ell}$, so

$$
\mu_{L}\left(w_{\ell}\right)=\mu^{\mathrm{W}}\left(w_{\ell}\right) \succ_{w_{\ell}} \mu_{\ell}\left(w_{\ell}\right) .
$$

The conditions of Definition 1 are therefore satisfied. 
The matching $\mu_{k^{1}+k^{2}+k^{3}}$ is equal to $\mu^{\mathrm{W}}$ or satisfies the assumptions of Lemma 1 . In the former case we are done, in the latter case we proceed as in the proof of Lemma 1 to complete the construction of the pairwise myopic-farsighted improving path leading to $\mu^{\mathrm{W}}$.

\section{Proof of Theorem 3.}

Suppose that $V \subset C$ is a pairwise myopic-farsighted stable set different from $\left\{\mu^{\mathrm{W}}\right\}$. It holds that $V \subset C \backslash\left\{\mu^{\mathrm{W}}\right\}$, since otherwise Lemma 1 implies that internal stability would be violated. Since $\mu^{\mathrm{W}} \notin V$, by external stability of $V$, there should be a pairwise myopic-farsighted improving path $\mu_{0}, \ldots, \mu_{L}$ from $\mu_{0}=\mu^{\mathrm{W}}$ to $\mu_{L} \in V$.

It holds that $\mu^{\mathrm{W}} \succeq_{w} \mu$ for all $\mu \in C$. Thus, since all women are farsighted, no woman $w \in W$ will form a match with a man $m \in M$ to move from $\mu_{0}=\mu^{\mathrm{W}}$ to $\mu_{1}=\mu_{0}+(m, w)$ in order to finish at $\mu \in C$ because she cannot strictly improve. For the same reason, no woman $w \in W$ will destroy a match with a man $m \in M$ to move from $\mu_{0}=\mu^{\mathrm{W}}$ to $\mu_{1}=\mu_{0}-(m, w)$ in order to finish at $\mu \in C$. The only remaining possibility is that a man destroys a link at $\mu_{0}=\mu^{\mathrm{W}}$. But since $\mu^{\mathrm{W}}$ is individually rational, this is not profitable. Hence, we conclude that there is no a pairwise myopic-farsighted improving path $\mu_{0}, \ldots, \mu_{L}$ from $\mu_{0}=\mu^{\mathrm{W}}$ to $\mu_{L} \in V$, and therefore $V \subset C \backslash\left\{\mu^{\mathrm{W}}\right\}$ cannot be a pairwise myopic-farsighted stable set.

\section{Proof of Theorem 4.}

Let $V \subset C$ be a pairwise myopic-farsighted stable set. By Theorem 3, the only possibility is $V=\left\{\mu^{\mathrm{W}}\right\}$. In order to satisfy external stability in Definition 2, it should hold that $\mu^{\mathrm{W}} \in h^{*}(\mu)$ for every $\mu \in \mathcal{M} \backslash\left\{\mu^{\mathrm{W}}\right\}$. However, notice that $\mu^{\mathrm{W}} \notin h^{*}(\bar{\mu})$ if $\bar{\mu} \in \mathcal{I} W$-dominates $\mu^{\mathrm{W}}$. Since all women are farsighted, no woman $w \in W$ will form a match with a man $m \in M$ to move from $\mu_{0}=\bar{\mu}$ to $\mu_{1}=\mu_{0}+(m, w)$ in order to finish at $\mu^{\mathrm{W}}$ because she cannot strictly improve. For the same reason, no woman $w \in W$ will destroy a match with a man $m \in M$ to move from $\mu_{0}=\bar{\mu}$ to $\mu_{1}=\mu_{0}-(m, w)$ in order to finish at $\mu^{\mathrm{W}}$. Also, since $\bar{\mu} \in \mathcal{I}$, no man can profitably deviate from $\mu_{0}=\bar{\mu}$ to $\mu_{1}=\mu_{0}-(m, w)$. Hence, we conclude that there is no a pairwise myopic-farsighted improving path $\mu_{0}, \ldots, \mu_{L}$ from $\mu_{0}=\bar{\mu}$ to $\mu_{L}=\mu^{\mathrm{W}}$, and therefore $\left\{\mu^{\mathrm{W}}\right\}$ cannot be a pairwise myopic-farsighted stable set. 
Lemma 3. Let $(M, W, F, \succ)$ be a marriage problem. Let $\mu_{0}, \ldots, \mu_{L}$ be a coalitional myopic-farsighted improving path from a matching $\mu \in \mathcal{M}$ to a matching $\mu^{\prime} \in \mathcal{M}$. If $i \in N \backslash F$ and $\mu(i) \succeq_{i} i$, then it holds that, for every $\ell \in\{0, \ldots, L\}, \mu_{\ell}(i) \succeq_{i} i$. If $i \in F$ and $\mu(i) \succeq_{i} i$, then it holds that $\mu^{\prime}(i) \succeq_{i} i$. If there is $\ell \in\{1, \ldots, L\}$ such that $i \in S_{\ell} \cap F$ and $\mu(i) \succeq_{i} i$, then it holds that $\mu^{\prime}(i) \succ_{i} i$.

Proof. For every $\ell \in\{0, \ldots, L-1\}$, let $S_{\ell} \in \mathcal{N}$ be the coalition that can enforce $\mu_{\ell+1}$ from $\mu_{\ell}$ and

$$
\begin{array}{ll}
\mu_{\ell+1}(i) \succ_{i} \mu_{\ell}(i) & \text { if } i \in S_{\ell} \backslash F, \\
\mu_{L}(i) \succ_{i} \mu_{\ell}(i) & \text { if } i \in S_{\ell} \cap F .
\end{array}
$$

We distinguish two cases.

Case 1. $i \in N \backslash F$.

We prove by induction that, for every $\ell=0, \ldots, L, \mu_{\ell}(i) \succeq_{i} i$. By assumption, $\mu_{0}(i) \succeq_{i} i$. Assume, for some $\ell=0, \ldots, L-1$, it holds that $\mu_{\ell}(i) \succeq_{i} i$. We show that $\mu_{\ell+1}(i) \succeq_{i} i$. We distinguish between three cases when moving from $\mu_{\ell}$ to $\mu_{\ell+1}$ :

Case i. $i$ creates a match with a player different from $\mu_{\ell}(i)$.

Case ii. $i$ becomes single.

Case iii. $i$ is not affected.

In Case i, it holds that $\mu_{\ell+1}(i) \succ_{i} \mu_{\ell}(i) \succeq_{i} i$, in Case ii, that $\mu_{\ell+1}(i)=i \succeq_{i} i$, and in Case iii, that $\mu_{\ell+1}(i)=\mu_{\ell}(i) \succeq_{i} i$. We have shown that $\mu_{\ell+1}(i) \succeq_{i} i$. It follows that $\mu^{\prime}(i)=\mu_{L}(i) \succeq_{i} i$.

Case 2. $i \in F$.

Suppose $i \succ_{i} \mu^{\prime}(i)$. Since $\mu_{0}(i) \succeq_{i} i$, it holds that $i \notin S_{0}$, so $\mu_{1}(i)=i$ (in case $\left.\mu_{0}(i) \in S_{0}\right)$ or $\mu_{1}(i)=\mu_{0}(i) \succeq_{i} i$. In both cases, it holds that $\mu_{1}(i) \succeq_{i} i$. Repeating this argument, it follows that $\mu^{\prime}(i)=\mu_{L}(i) \succeq_{i} i$, leading to a contradiction. Consequently, it holds that $\mu^{\prime}(i)=\mu_{L}(i) \succeq_{i} i$.

Assume $\mu_{L}(i)=i$. Since $\mu_{0}(i) \succeq_{i} i$, it follows that $i \notin S_{0}$ and $\mu_{1}(i)=\mu_{0}(i)$ or $\mu_{1}(i)=i$, so $\mu_{1}(i) \succeq_{i} i$. By repeating this argument, we find that, for every $\ell \in\{0, \ldots, L-1\}, \mu_{\ell}(i) \succeq_{i} i$. Since moves by coalitions involve strict improvement, we have that, for every $\ell \in\{0, \ldots, L-1\}, i \in N \backslash S_{\ell}$. It follows that if there is $\ell \in\{0, \ldots, L-1\}$ such that $i \in S_{\ell}$, then $\mu_{L}(i) \succ_{i} i$. 


\section{Proof of Theorem 5.}

Let some $\mu \in \mathcal{I}$ be given.

Obviously, it holds that $h^{*}(\mu) \subset g^{*}(\mu)$.

We show now that $g^{*}(\mu) \subset h^{*}(\mu)$. Let $\mu^{\prime} \in g^{*}(\mu)$ be given. There is a finite sequence of distinct matchings $\mu_{0}, \ldots, \mu_{L}$ with $\mu_{0}=\mu$ and $\mu_{L}=\mu^{\prime}$ such that, for every $\ell \in\{0, \ldots, L-1\}$, there is a coalition $S_{\ell} \in \mathcal{N}$ that can enforce $\mu_{\ell+1}$ from $\mu_{\ell}$ and

$$
\begin{array}{ll}
\mu_{\ell+1}(i) \succ_{i} \mu_{\ell}(i), & \text { if } i \in S_{\ell} \backslash F, \\
\mu_{L}(i) \succ_{i} \mu_{\ell}(i), & \text { if } i \in S_{\ell} \cap F .
\end{array}
$$

Let some $\ell \in\{0, \ldots, L-1\}$ be given. We define

$$
\begin{aligned}
& D_{\ell}^{0}=\left\{m \in S_{\ell} \mid \mu_{\ell}(m) \in W, \mu_{\ell+1}(m)=m, \text { and } \mu_{\ell+1}\left(\mu_{\ell}(m)\right)=\mu_{\ell}(m)\right\}, \\
& D_{\ell}^{1}=\left\{w \in S_{\ell} \mid \mu_{\ell}(w) \in M \backslash D_{\ell}^{0}, \mu_{\ell+1}(w)=w, \text { and } \mu_{\ell+1}\left(\mu_{\ell}(w)\right)=\mu_{\ell}(w)\right\},
\end{aligned}
$$

as the men in $S_{\ell}$ who at $\mu_{\ell}$ destroy their match with a woman and the women in $S_{\ell}$ who at $\mu_{\ell}$ destroy their match with a man outside $D_{\ell}^{0}$. The reason to exclude men in $D_{\ell}^{0}$ is to avoid double counting. Let $D_{\ell}=D_{\ell}^{0} \cup D_{\ell}^{1}$. The cardinality of $D_{\ell}$ is denoted by $d_{\ell}$. We will have the players in $D_{\ell}$ successively destroying their matches when constructing a pairwise myopic-farsighted improving path from $\mu_{\ell}$ to $\mu_{\ell+1}$. By Lemma 3 it holds for every $i \in N \backslash F$ that $\mu_{\ell}(i) \succeq_{i} i$, so myopic players in $S_{\ell}$ are never destroying matches when going from $\mu_{\ell}$ to $\mu_{\ell+1}$, and therefore $D_{\ell} \subset F$. We define the set $A_{\ell}=\left\{m \in S_{\ell} \mid \mu_{\ell}(m) \neq \mu_{\ell+1}(m) \in W\right\}$ as the set of men that form a match when going from $\mu_{\ell}$ to $\mu_{\ell+1}$. Note that enforceability of $\mu_{\ell+1}$ implies that $\mu_{\ell+1}(m) \in S_{\ell}$. The requirement $\mu_{\ell}(m) \neq \mu_{\ell+1}(m)$ is added to ensure that $m$ does not simply keep his partner at $\mu_{\ell}$. The cardinality of $A_{\ell}$ is denoted by $a_{\ell}$.

Take an arbitrary order of the individuals in $D_{\ell}$, say $i_{0}, \ldots, i_{d_{\ell}-1}$, and an arbitrary order of the men in $A_{\ell}$, say $m_{0}, \ldots, m_{a_{\ell}-1}$. We define $\nu_{\ell, 0}=\mu_{\ell}$. For $k \in\left\{0, \ldots, d_{\ell}-1\right\}$, we define the matching $\nu_{\ell, k+1}=\nu_{\ell, k}-\left(i_{k}, \mu_{\ell}\left(i_{k}\right)\right)$. For $k \in\left\{0, \ldots, a_{\ell}-1\right\}$, we define the matching $\nu_{\ell, d_{\ell}+k+1}=\nu_{\ell, d_{\ell}+k}+\left(m_{k}, \mu_{\ell+1}\left(m_{k}\right)\right)$.

Consider the sequence of distinct matchings

$$
\nu_{0,0}, \ldots, \nu_{0, d_{0}+a_{0}-1}, \nu_{1,0}, \ldots, \nu_{L-1,0}, \ldots, \nu_{L-1, d_{L-1}+a_{L-1}} .
$$

Notice that $\nu_{L-1, d_{L-1}+a_{L-1}}=\mu_{L}$. We argue that this sequence is a pairwise myopicfarsighted improving path from $\nu_{0,0}=\mu$ to $\nu_{L-1, d_{L-1}+a_{L-1}}=\mu^{\prime}$. 
Let some $\ell \in\{0, \ldots, L-1\}$ and some $k \in\left\{0, \ldots, d_{\ell}-1\right\}$ be given. It holds that $\nu_{\ell, k+1}=\nu_{\ell, k}-\left(i_{k}, \mu_{\ell}\left(i_{k}\right)\right)$. Since $i_{k} \in D_{\ell}$, we have that $i_{k} \in F$. It holds that $\nu_{\ell, k}\left(i_{k}\right)=\mu_{\ell}\left(i_{k}\right) \prec_{i_{k}} \mu_{L}\left(i_{k}\right)$, where the last step uses that $i_{k} \in S_{\ell}$.

Let some $\ell \in\{0, \ldots, L-1\}$ and some $k \in\left\{0, \ldots, a_{\ell}-1\right\}$ be given. For $w_{k}=$ $\mu_{\ell+1}\left(m_{k}\right)$, it holds that $\nu_{\ell, d_{\ell}+k+1}=\nu_{\ell, d_{\ell}+k}+\left(m_{k}, w_{k}\right)$. First, consider the case where $m_{k} \in F$. We have $\mu_{L}\left(m_{k}\right) \succ_{m_{k}} \mu_{\ell}\left(m_{k}\right)$ and, by Lemma 3, $\mu_{L}\left(m_{k}\right) \succ_{m_{k}} m_{k}$. It also holds that $\nu_{\ell, d_{\ell}+k}\left(m_{k}\right)=\mu_{\ell}\left(m_{k}\right)$ or $\nu_{\ell, d_{\ell}+k}\left(m_{k}\right)=m_{k}$. In both cases, we find that $\mu_{L}\left(m_{k}\right) \succ_{m_{k}} \nu_{\ell, d_{\ell}+k}\left(m_{k}\right)$. The same argument can be used to show that in case $w_{k} \in F$ it holds that $\mu_{L}\left(w_{k}\right) \succ_{w_{k}} \nu_{\ell, d_{\ell}+k}\left(w_{k}\right)$. Second, consider the case where $m_{k} \in M \backslash F$. Using Lemma 3, we have $\mu_{\ell+1}\left(m_{k}\right) \succ_{m_{k}} \mu_{\ell}\left(m_{k}\right) \succeq_{m_{k}} m_{k}$. It also holds that $\nu_{\ell, d_{\ell}+k+1}\left(m_{k}\right)=\mu_{\ell+1}\left(m_{k}\right)$ and $\nu_{\ell, d_{\ell}+k}\left(m_{k}\right)=\mu_{\ell}\left(m_{k}\right)$ or $\nu_{\ell, d_{\ell}+k}\left(m_{k}\right)=m_{k}$. In both cases we find that $\nu_{\ell, d_{\ell+k+1}}\left(m_{k}\right) \succ_{m_{k}} \nu_{\ell, d_{\ell}+k}\left(m_{k}\right)$. The same argument can be used to show that in case $w_{k} \in W \backslash F$, then $\nu_{\ell, d_{\ell+k+1}}\left(w_{k}\right) \succ_{w_{k}} \nu_{\ell, d_{\ell}+k}\left(w_{k}\right)$.

We have shown that $\mu^{\prime} \in h^{*}(\mu)$.

Lemma 4. Let $(M, W, F, \succ)$ be a marriage problem. Let $V$ be a pairwise myopicfarsighted stable set. It holds that $V \subset \mathcal{I}$.

Proof. Suppose $\mu \in V$ is not individually rational. Then there is $m \in M$ and $w \in W$ such that $\mu(m)=w, m \succ_{m} w$ or $w \succ_{w} m$. Without loss of generality, assume $m \succ_{m} w$. We show that $\mu \notin h^{*}(\mu-(m, w))$ by distinguishing two cases.

Case 1. $m \in M \backslash F$.

Suppose $\mu_{0}, \ldots, \mu_{L}$ is a pairwise myopic-farsighted improving path from $\mu_{0}=\mu-$ $(m, w)$ to $\mu_{L}=\mu$. We prove by induction that, for every $\ell=0, \ldots, L, \mu_{\ell}(m) \succeq_{m} m$. Since $\mu_{0}(m)=m$, it clearly holds that $\mu_{0}(m) \succeq_{m} m$. Assume, for some $\ell=0, \ldots, L-$ 1 , it holds that $\mu_{\ell}(m) \succeq_{m} m$. We show that $\mu_{\ell+1}(m) \succeq_{m} m$. We distinguish between three cases when moving from $\mu_{\ell}$ to $\mu_{\ell+1}:$ a. $m$ creates a match with a woman different from $\mu_{\ell}(m)$; b. $m$ becomes single; c. $m$ is not affected. In Case a it holds that $\mu_{\ell+1}(m) \succ_{m} \mu_{\ell}(m) \succeq_{m} m$, in Case b that $\mu_{\ell+1}(m)=m \succeq_{m} m$, and in Case $\mathrm{c}$ that $\mu_{\ell+1}(m)=\mu_{\ell}(m) \succeq_{m} m$. We have shown that $\mu_{\ell+1}(m) \succeq_{m} m$. It follows that $\mu_{L}(m) \succeq_{m} m$, yielding a contradiction to $m \succ_{m} \mu_{L}(m)=w$. Consequently, there is no pairwise myopic-farsighted improving path from $\mu-(m, w)$ to $\mu$ if $m \in M \backslash F$.

Case 2. $m \in F$.

Suppose $\mu_{0}, \ldots, \mu_{L}$ is a pairwise myopic-farsighted improving path from $\mu_{0}=\mu-$ 
$(m, w)$ to $\mu_{L}=\mu$. Since $\mu-(m, w) \succ_{m} \mu$, it is not possible that $m$ is involved in the formation of a match with a woman when moving from $\mu_{0}$ to $\mu_{1}$, so $\mu_{1}(m)=m$. By repeating this argument, we find that $\mu_{1}(m)=\cdots=\mu_{L}(m)=m$, a contradiction to $\mu_{L}(m)=w$. Consequently, there is no pairwise myopic-farsighted improving path from $\mu-(m, w)$ to $\mu$ if $m \in F$.

Combining Cases 1 and 2, we have shown that $\mu \notin h^{*}(\mu-(m, w))$.

On the other hand, $\mu-(m, w) \in h^{*}(\mu)$, so by $\mu \in V$ and internal stability of $V$ it follows that $\mu-(m, w) \notin V$. By external stability of $V$ it holds that there is $\mu^{\prime} \in h^{*}(\mu-(m, w))$ such that $\mu^{\prime} \in V$. Since $\mu \notin h^{*}(\mu-(m, w))$, we have $\mu^{\prime} \neq \mu$.

Let $\mu_{1}, \ldots, \mu_{L}$ with $\mu_{1}=\mu-(m, w)$ and $\mu_{L}=\mu^{\prime}$ be a pairwise myopic-farsighted improving path from $\mu-(m, w)$ to $\mu^{\prime}$. We distinguish between two cases.

Case 1. $m \in M \backslash F$.

It holds that $\mu-(m, w) \succ_{m} \mu$. Therefore, it follows that $\mu_{0}, \mu_{1}, \ldots, \mu_{L}$ with $\mu_{0}=\mu$ is a pairwise myopic-farsighted improving path from $\mu$ to $\mu^{\prime}$ and therefore $\mu^{\prime} \in h^{*}(\mu)$. This contradicts the fact that $V$ is internally stable.

Case 2. $m \in F$.

Since $\mu^{\prime} \in h^{*}(\mu-(m, w))$ and $m \in F$, it follows as before that $\mu^{\prime}(m) \succeq_{m} m$. Therefore it holds that $\mu_{0}, \mu_{1}, \ldots, \mu_{L}$ with $\mu_{0}=\mu$ is a pairwise myopic-farsighted improving path from $\mu$ to $\mu^{\prime}$ and therefore $\mu^{\prime} \in h^{*}(\mu)$. This contradicts the fact that $V$ is internally stable.

\section{Proof of Theorem 6.}

Let $V$ be a pairwise myopic-farsighted stable set.

For every $\mu, \mu^{\prime} \in V$, it holds that $\mu^{\prime} \notin h^{*}(\mu)$. By Lemma $4, \mu$ is individually rational, so by Theorem 5 we have that $\mu^{\prime} \notin g^{*}(\mu)$. This shows that $V$ satisfies internal stability as defined in Definition 6 .

For every $\mu \in \mathcal{M} \backslash V$, it holds that $h^{*}(\mu) \cap V \neq \emptyset$. Since $h^{*}(\mu) \subset g^{*}(\mu)$, it follows that $g^{*}(\mu) \cap V \neq \emptyset$. This shows that $V$ satisfies external stability as defined in Definition 6.

We conclude that $V$ is a coalitional myopic-farsighted stable set.

Let $V$ be a coalitional myopic-farsighted stable set.

For every $\mu, \mu^{\prime} \in V$, it holds that $\mu^{\prime} \notin g^{*}(\mu)$. Since $h^{*}(\mu) \subset g^{*}(\mu)$, it follows that $\mu^{\prime} \notin h^{*}(\mu)$. This shows that $V$ satisfies internal stability as defined in Definition 2.

We now show that every matching in $V$ is individually rational. Suppose $\mu \in V$ is not individually rational. Then there is $m \in M$ and $w \in W$ such that $\mu(m)=w$, 
$m \succ_{m} w$ or $w \succ_{w} m$. Without loss of generality, assume $m \succ_{m} w$. It follows from Lemma 3 that $\mu \notin g^{*}(\mu-(m, w))$. On the other hand, $\mu-(m, w) \in g^{*}(\mu)$, so by $\mu \in V$ and internal stability of $V$ as defined in Definition 6 it follows that $\mu-(m, w) \notin V$. By external stability of $V$ as defined in Definition 6 it holds that there is $\mu^{\prime} \in g^{*}(\mu-(m, w))$ such that $\mu^{\prime} \in V$. Since $\mu \notin g^{*}(\mu-(m, w))$, we have $\mu^{\prime} \neq \mu$.

Let $\mu_{1}, \ldots, \mu_{L}$ with $\mu_{1}=\mu-(m, w)$ and $\mu_{L}=\mu^{\prime}$ be a coalitional myopicfarsighted improving path from $\mu-(m, w)$ to $\mu^{\prime}$. We distinguish between two cases.

Case 1. $m \in M \backslash F$.

It holds that $\mu-(m, w) \succ_{m} \mu$. Therefore, it follows that $\mu_{0}, \mu_{1}, \ldots, \mu_{L}$ with $\mu_{0}=\mu$ is a coalitional myopic-farsighted improving path from $\mu$ to $\mu^{\prime}$ and therefore $\mu^{\prime} \in g^{*}(\mu)$. This contradicts the fact that $V$ is internally stable as defined in Definition 6 .

Case 2. $m \in F$.

Since $\mu^{\prime} \in g^{*}(\mu-(m, w))$ and $m \in F$, it follows from Lemma 3 that $\mu^{\prime}(m) \succeq_{m} m$. Therefore it holds that $\mu_{0}, \mu_{1}, \ldots, \mu_{L}$ with $\mu_{0}=\mu$ is a coalitional myopic-farsighted improving path from $\mu$ to $\mu^{\prime}$ and therefore $\mu^{\prime} \in g^{*}(\mu)$. This contradicts the fact that $V$ is internally stable as defined in Definition 6.

Consequently, every matching in $V$ is individually rational.

Let some $\mu \in \mathcal{M} \backslash V$ be given. We distinguish between two cases.

Case 1. $\mu \in \mathcal{I}$.

Since $V$ is a coalitional myopic-farsighted stable set, it holds that $g^{*}(\mu) \cap V \neq \emptyset$. By Theorem 5 it holds that $h^{*}(\mu)=g^{*}(\mu)$, so $h^{*}(\mu) \cap V \neq \emptyset$.

Case 2. $\mu \in \mathcal{M} \backslash \mathcal{I}$.

We define

$$
\begin{aligned}
D^{0} & =\left\{m \in M \mid m \succ_{m} \mu(m)\right\}, \\
D^{1} & =\left\{w \in W \mid w \succ_{w} \mu(w) \text { and } \mu(w) \notin D^{0}\right\},
\end{aligned}
$$

as the set of men that prefer to be single over their match at $\mu$ and the set of women that are not married to a man in $D^{0}$ and prefer to be single over their match at $\mu$. Let $D=D^{0} \cup D^{1}$ and denote the cardinality of $D$ by $d \geq 1$. Take an arbitrary order of the individuals in $D$, say $i_{0}, \ldots, i_{d}-1$. Define $\mu_{0}=\mu$. For $\ell \in\{0, \ldots, d-1\}$, we define the matching $\mu_{\ell+1}=\mu_{\ell}-\left(i_{\ell}, \mu\left(i_{\ell}\right)\right)$. We distinguish two subcases.

Subcase 2.1. $\mu_{d} \in V$.

We show that $\mu_{0}, \ldots, \mu_{d}$ is a pairwise myopic-farsighted improving path from $\mu_{0}=\mu$ 
to $\mu_{d} \in V$. Let some $\ell \in\{0, \ldots, d-1\}$ be given. If $i_{\ell} \in N \backslash F$, then $\mu_{\ell+1}\left(i_{\ell}\right)=i_{\ell} \succ_{i_{\ell}}$ $\mu\left(i_{\ell}\right)=\mu_{\ell}\left(i_{\ell}\right)$. If $i_{\ell} \in F$, then $\mu_{d}\left(i_{\ell}\right)=i_{\ell} \succ_{i_{\ell}} \mu\left(i_{\ell}\right)=\mu_{\ell}\left(i_{\ell}\right)$.

Subcase $2.2 \mu_{d} \notin V$.

If $\mu_{d} \notin V$, then let $\mu^{\prime} \in V$ be such that $\mu^{\prime} \in g^{*}\left(\mu_{d}\right)$. The existence of $\mu^{\prime}$ follows from the external stability of $V$ according to Definition 6. Since $\mu_{d}$ is individually rational by construction, we can use Theorem 5 to conclude that $h^{*}\left(\mu_{d}\right)=g^{*}\left(\mu_{d}\right)$, so $\mu^{\prime} \in h^{*}\left(\mu_{d}\right)$. Let $\mu_{d}, \mu_{d+1}, \ldots, \mu_{d+L}$ be a pairwise myopic-farsighted improving path from $\mu_{d}$ to $\mu_{d+L}=\mu^{\prime}$. We show next that $\mu_{0}, \mu_{1}, \ldots, \mu_{d+L}$ is a pairwise myopicfarsighted improving path from $\mu_{0}$ to $\mu_{d+L}$.

Let some $\ell \in\{0, \ldots, d-1\}$ be given. If $i_{\ell} \in N \backslash F$, then $\mu_{\ell+1}\left(i_{\ell}\right)=i_{\ell} \succ_{i_{\ell}} \mu\left(i_{\ell}\right)=$ $\mu_{\ell}\left(i_{\ell}\right)$. If $i_{\ell} \in F$, then $\mu_{d+L}\left(i_{\ell}\right) \succeq_{i_{\ell}} i_{\ell} \succ_{i_{\ell}} \mu\left(i_{\ell}\right)=\mu_{\ell}\left(i_{\ell}\right)$, where the first step uses the fact that $\mu^{\prime}$ belongs to $V$, so is individually rational. For $\ell \in\{d, \ldots, d+L-$ $1\}$, the moving players make a strict improvement in the appropriate sense since $\mu_{d}, \mu_{d+1}, \ldots, \mu_{d+L}$ is a pairwise myopic-farsighted improving path from $\mu_{d}$ to $\mu_{d+L}$.

We have shown that $h^{*}(\mu) \cap V \neq \emptyset$ and therefore that $V$ satisfies external stability as defined in Definition 2.

We conclude that $V$ is a pairwise myopic-farsighted stable set.

\section{References}

Anesi, V. (2010), "Noncooperative Foundations of Stable Sets in Voting Games", Games and Economic Behavior, 70, 488-493.

Banerjee, S., H. Konishi and T. Sönmez (2001), "Core in a Simple Coalition Formation Game", Social Choice and Welfare 18, 135-153.

Bloch, F. (1996), "Sequential Formation of Coalitions in Games with Externalities and Fixed Payoff Division", Games and Economic Behavior 14, 90-123.

Bloch, F., and A. van den Nouweland (2020), "Farsighted Stability with Heterogeneous Expectations", Games and Economic Behavior 121, 32-54.

Chwe, M. S.-Y. (1994), "Farsighted Coalitional Stability", Journal of Economic Theory 63, 299-325. 
Demuynck, T., P.J.J. Herings, R.D. Saulle, and C. Seel (2019), "The Myopic Stable Set for Social Environments", Econometrica 87, 111-138.

Diamantoudi, E., and L. Xue (2003), "Farsighted Stability in Hedonic Games", Social Choice and Welfare 21, 39-61.

Dutta, B., and H. Vartiainen (2020), "Coalition Formation and History Dependence", Theoretical Economics, 15, 159-197.

Dutta, B., and R. Vohra (2017), "Rational Expectations and Farsighted Stability", Theoretical Economics, 12, 1191-1227.

Ehlers, L. (2007), "Von Neumann-Morgenstern Stable Sets in Matching Problems", Journal of Economic Theory 134, 537-547.

Gale, D., and L.S. Shapley (1962), "College Admissions and the Stability of Marriage", American Mathematical Monthly 69, 9-15.

Harsanyi, J.C. (1974), "An Equilibrium-Point Interpretation of Stable Sets and a Proposed Alternative Definition", Management Science 20, 1472-1495.

Herings, P.J.J., A. Mauleon and V. Vannetelbosch (2004), "Rationalizability for Social Environments", Games and Economic Behavior 49, 135-156.

Herings, P.J.J., A. Mauleon and V. Vannetelbosch (2009), "Farsightedly Stable Networks", Games and Economic Behavior 67, 526-541.

Herings, P.J.J., A. Mauleon and V. Vannetelbosch (2017), "Stable Sets in Matching Problems with Coalitional Sovereignty and Path Dominance", Journal of Mathematical Economics 71, 14-19.

Herings, P.J.J., A. Mauleon, and V. Vannetelbosch (2020), "Matching with Myopic and Farsighted Players", Journal of Economic Theory 190, 105125.

Kimja, M. (2020) "Equilibrium Coalitional Behavior", Theoretical Economics 15, 669-714.

Knuth, D.E. (1976), Marriages Stables, Les Presses de l'Université de Montreal, Montreal. 
Konishi, H., and D. Ray (2003), "Coalition Formation as a Dynamic Process", Journal of Economic Theory, 110, 1-41.

Mauleon, A., V. Vannetelbosch and W. Vergote (2011), "Von Neumann-Morgenstern Farsightedly Stable Sets in Two-sided Matching", Theoretical Economics 6, 499-521.

Page, F.H., Jr. and M. Wooders (2009), "Strategic Basins of Attraction, the Path Dominance Core, and Network Formation Games", Games and Economic Behavior 66, 462-487.

Ray, D. and R. Vohra (2015), "The Farsighted Stable Set", Econometrica 83, 977-1011.

Ray, D., and R. Vohra (2019), "Maximality in the Farsighted Stable Set", Econometrica 87, 1763-1779.

Roth, A.E. and M.A.O. Sotomayor (1990), Two-Sided Matching, A Study in Game-Theoretic Modeling and Analysis, Econometric Society Monographs No. 18, Cambridge University Press, Cambridge.

Vartiainen, H. (2011), "Dynamic Coalitional Equilibrium", Journal of Economic Theory 146, 672-698.

Wako, J. (2010), "A Polynomial-Time Algorithm to Find von Neumann-Morgenstern Stable Matchings in Marriage Games", Algorithmica 58, 188-220.

Xue, L. (1998), "Coalitional Stability under Perfect Foresight", Economic Theory $11,603-627$. 